\title{
A comparison of the chemical compounds in single- and co-boiled Zhi-Zi-Da- Huang decoctions with UPLC-qTOF-MS/MS and UPLC-DAD
}

\author{
Mengyu Xia \\ Institute of Chinese Materia Medica, China Academy of Chinese Medical Sciences \\ Xue Zhang \\ Chinese Academy of Medical Sciences \& Peking Union Medical College Hospital of Skin Diseases and Institute of Dermatology \\ Hongwei Wu \\ Institute of Chinese Materia Medica, China Academy of Chinese Medical Sciences \\ Yun Wang \\ Institute of Chinese Materia Medica, China Academy of Chinese Medical Sciences \\ Cun Zhang ( $\sim$ zhc95@163.com) \\ Institute of Chinese Materia Medica, China Academy of Chinese Medical Sciences https://orcid.org/0000-0001-8738-7698
}

\section{Research}

Keywords: Zhi-Zi-Da-Huang decoction, chemical substances, single-boiled, co-boiled, UPLC-qTOF-MS/MS, UPLC-DAD

Posted Date: August 18th, 2020

DOI: https://doi.org/10.21203/rs.3.rs-56884/v1

License: @ (1) This work is licensed under a Creative Commons Attribution 4.0 International License. Read Full License 


\section{Abstract \\ Background}

Zhi-Zi-Da-Huang decoction (ZZDHD) is a famous Traditional Chinese Medicine decoction due to its therapeutic effects on clinical hepatobiliary disorders. ZZDHD is composed of Gardeniae Fructus, Rhei Radix et Rhizoma, Fructus Aurantii Immaturus, and Sojae Semen Praeparatum. With the development of current technology, dispensing granules have been widely used for convenience. However, limited research has been conducted to determine differences in the chemical compounds between dispensing granules and traditional decoction.

\section{Methods}

A strategy based on UPLC-qTOF-MS/MS and UPLC-DAD was established to quantitatively and qualitatively analyze the chemical compounds present in single- and co-boiled ZZDHD. First, we utilized UPLC-qTOF-MS/MS to identify the compounds in single- and co-boiled ZZDHD. Then, 15 compounds were quantitatively analyzed in ZZDHD by UPLC-DAD. Finally, fingerprint and chemometric analyses were adopted to evaluate the difference between single- and co-boiled ZZDHD.

\section{Results}

One hundred and forty-seven compounds were authenticated or determined according to their retention behaviors, mass mensuration, and characteristic fragment ions. In addition, 15 analytes of single- and co-boiled ZZDHD indicated a significant difference in the content of many compounds, including geniposide (G2), emodin, chrysophanol, daidzin, and crocin-I. The similarities of single and co-boiled ZZDHD were higher than 0.99, as evaluated by fingerprinting; 30 common peaks were evaluated by the chemometric analysis, which found 13 important variable values (VIP > 1 ) including C3, A3, crocetin, daidzin, C5, physcion, G2, genipin-1-0- $\beta$-D-gentiobioside (G1), chrysophanol, emodin, A2, A5, and C6.

\section{Conclusions}

This study provided an experimental basis for explaining the rationality of single-and co-boiled ZZDHD, and a study model for comparing the chemical substances between traditional decoctions and dispensing granules.

\section{Background}

As combinations of multiple compounds, Traditional Chinese Medicine decoctions are the oldest and most commonly used treatments for certain diseases. During the decoction process, chemical compounds may interact with each other due to the chemical components present in each herb. For example, large changes in the dissolution rate, generation of new chemical compounds, and changes in pharmacological effects and clinical efficacy might occur during decoction[1-3]. Dispensing granules in Chinese medicine consist of individual Chinese medicinals (e.g., plants) obtained by water extraction, concentration, drying, and granulation. Dispensing granules originated from compound granules produced in Japan in the 1970s, which were subsequently used in South Korea and Taiwan, China[4]. Compared with traditional decoctions, dispensing granules are considered to be faster, simpler, and more convenient for clinical application[5]; however, there is a controversy about the effect of co-decoction of dispensing granules that is reflected in the literature. Many studies have reported that the clinical effects of dispensing granules are equivalent to or better than the effects of traditional decoctions[6, 7], indicating that dispensing granules could replace traditional decoctions; however, there are some issues that need to be considered in the development of dispensing granules. Specifically, it is important to determine whether the production process is consistent, whether a quality control standard has been established, and whether significant differences exist in the main active components, pharmacodynamics, and clinical efficacy. However, not all traditional decoctions can be replaced with dispensing granules. Over the past decades, a few studies have revealed differences in the content of marker compounds and chemical composition between dispensing granules and traditional decoctions[8-11]. Therefore, it is essential to evaluate the chemical consistency between traditional decoctions and dispensing granules of Traditional Chinese Medicine recipes by means of modern analytical methods and technologies.

Zhi-Zi-Da-Huang decoction (ZZDHD) is a widely used Traditional Chinese Medicine decoction that contains a combination of Gardeniae Fructus (Zhi Zi, in Mandarin Chinese, ZZ), Rhei Radix et Rhizoma (Da Huang, in Mandarin Chinese, DH), Fructus Aurantii Immaturus (Zhi Shi, in Mandarin Chinese, ZS) and Sojae Semen Praeparatum (Dan Dou Chi, in Mandarin Chinese, DDC). The fruit of Gardenia jasminoides Ellis is included in the Chinese Pharmacopoeia under the item of ZZ. Similarly, the rhizomes and roots of Rheum palmatum L., Rheum tanguticum Maxim. ex Balf., and Rheum officinale Baill. are recorded under the item of DH. ZS derives from immature fruits of Citrus aurantium L. and Citrus sinensis (L.) Osbeck. DDC is a fermented product obtained from the ripe seed of Glycine $\max$ (L.) Merr. As a classical prescription for clearing heat and eliminating dampness and jaundice, ZZDHD was originally recorded in book of 'Jin Gui Yao Lve' by the physician Zhang Zhongjing (in Eastern Han Dynasty of ancient China). With the theory of Traditional Chinese Medicine, the published paper showed that ZZDHT was used in various diseases caused by heat and dampness. For example, in 80 cases of angina pectoris which was caused by heat stagnation and blood stasis, the effective rate of ZZDHT was 92.5\%[12]. ZZDHD is appropriate for the treatment of acute or chronic hepatitis, alcoholic liver disease (ALD), jaundice and cholestasis[13-16]. Jaundice was closely related to the disorder of liver in the theory of TCM, and the clinical observation proved that ZZDHT had a good effect on jaundice hepatitis patients[17]. A previous pharmacological study also demonstrated that ZZDHD exhibited a significant hepatoprotective activity by reversing histopathological changes and the biochemical parameters, like aspartate transaminase (AST), alanine transferase (ALT), reduced glutathione (GSH), malondialdehyde (MDA) and superoxide dismutase (SOD)[18]. Another investigation indicated that ZZDHD exerted a hepatoprotective effect on ANIT-induced liver injury with cholestasis by reversing the changes in bile flow, the serum and hepatic enzymes, and histopathology 
of the liver tissue, and the mechanism of this activity is possibly related to its antioxidant properties[14]. ZZDHD has complex chemical compounds, and its main active ingredients include iridoid glucosides and crocins in ZZ, flavonoids in ZS and DDC, and anthraquinone in DH[19-24]. In order to facilitate clinical applications, Traditional Chinese Medicines have been developed from the decoction into a dispensing granule. However, study on the traditional decoction and dispensing granule of ZZDHD is limited. There is currently controversy about the co-boiled Zhi-Zi-Da-Huang decoction (CB, all plants were boiled together) and the single-boiled Zhi-Zi-Da-Huang decoction (SB, each plant was boiled separately).

Recently, due to the rapid development of multi combination and mixed mass spectrometry (MS) technology, an increasing number of complex Traditional Chinese Medicine decoctions have been characterized globally. Qualitative and quantitative analyses were performed by multi-wavelength ultra-highperformance liquid chromatography (UHPLC) with diode array detection detector and electrospray ionization with quadrupole time-of-flight-tandem mass spectrometry $[9,25]$. These technologies have been used to evaluate the consistency of chemical compounds between traditional decoctions and dispensing granules. Liu utilized the method of ultra-performance liquid chromatography with diode array detection coupled to electrospray ionization and quadrupole time-of-flight mass spectrometry (UPLC-DAD-ESI-qTOF-MS) to distinguish the differences in chemical composition between the combined and separated decoctions of Shen-Shuai-Ning granules[9]. Shang proposed and validated ultra-performance liquid chromatography-quadrupole time-of-flight mass spectrometry (UPLC-qTOF-MS/MS) method to rapidly analyze chemical changes between traditional decoction and dispensing granules of San-Ao-Tang[10]. Thus, detecting and identifying more chemical information of traditional decoctions by the methods is worthy of consideration.

The primary goal of the present study was to compare the chemical compounds from single- and double-boiled Zhi-Zi-Da-Huang decoctions. First, chemical compounds were rapidly analyzed by UPLC-qTOF-MS/MS, and the significantly changed components were identified by comparing their mass spectra with the LC-MS/MS library. Second, 15 compounds in ZZDHD were quantified by UPLC-DAD. Finally, based on the qualitative analysis of UPLC fingerprints for comparison of various samples, several chemometric analyses, including principal component analysis (PCA), partial least-squares discrimination analysis (PLS-DA), and hierarchical cluster analysis (HCA), were achieved to further evaluate the differences in quality of these ZZDHD samples. The results obtained from this study could provide a meaningful basis for explaining the rationality of single- and co-boiled ZZDHD.

\section{Materials And Methods}

\subsection{Chemicals and reagents}

Acetonitrile was of HPLC grade (Merck, Darmstadt, Germany). Formic acid and methanol were of analytical grade. Water was purchased from Hangzhou Wahaha Company. Genipin-1-O- $\beta$-D-gentiobioside (G1) and geniposide (G2) standards were isolated and purified from Gardenia jasminoides in our laboratory. Crocin-I and crocetin were purchased from the Chengdu Must Biotechnology Co., Ltd. (Chengdu, China) and Chengdu Chroma-Biotechnology Co., Ltd. (Chengdu, China), respectively. Narirutin, naringin, hesperidin, neohesperidin, rhein, emodin, chrysophanol, physcion, daidzin, daidzein, and genistein standards were purchased from the Chengdu Alfa Biotechnology Co., Ltd. (Chengdu, China). The purity of 15 compounds was over $98 \%$ as determined by HPLC, and their chemical structures are shown in Fig. 1.

Gardeniae Fructus (ZZ), Rhei Radix et Rhizoma (DH), Aurantti Fructus Immaturus (ZS), and Sojae Semen Praeparatum (DDC) were purchased from Hebei Baicaokangshen Pharmaceutical Co., Ltd. and verified by Professor Yongqing Xiao of the Institute of Chinese Materia Medica China Academy of Chinese Medical Sciences.

\subsection{Preparation of standard and sample solutions}

\subsubsection{Preparation of standard solutions}

Standards of fifteen compounds were accurately weighed and separately dissolved in methanol to obtain the stock solution. One mixed stock solution of G2, $\mathrm{G} 1$, naringin, and neohesperidin was obtained with a concentration of $4,430 \mu \mathrm{g} / \mathrm{mL}, 1,380 \mu \mathrm{g} / \mathrm{mL}, 4,520 \mu \mathrm{g} / \mathrm{mL}$, and 3,700 $\mu \mathrm{g} / \mathrm{mL}$, respectively. The other mixed stock solution containing daidzin, daidzein, genistein, narirutin, hesperidin, crocin-l, rhein, crocetin, emodin, chrysophanol, and physcion was prepared with concentrations of $64.50 \mu \mathrm{g} / \mathrm{mL}, 41.08 \mu \mathrm{g} / \mathrm{mL}, 39.284 \mu \mathrm{g} / \mathrm{mL}, 514 \mu \mathrm{g} / \mathrm{mL}, 814 \mu \mathrm{g} / \mathrm{mL}, 380 \mu \mathrm{g} / \mathrm{mL}, 71 \mu \mathrm{g} / \mathrm{mL}, 1.502 \mu \mathrm{g} / \mathrm{mL}, 12.45 \mu \mathrm{g} / \mathrm{mL}, 6.645 \mu \mathrm{g} / \mathrm{mL}$, and $1.664 \mu \mathrm{g} / \mathrm{mL}$, respectively. The stock solutions were all stored in a refrigerator at $4{ }^{\circ} \mathrm{C}$ for further analysis.

\subsubsection{Preparation of the co-boiled Zhi-Zi-Da-Huang decoction solutions (CB)}

The four herbs in ZZDHD, which had the following contents: $18.0 \mathrm{~g}$ of ZZ, $24.0 \mathrm{~g}$ of ZS, $20.0 \mathrm{~g}$ of DDC, and $6.0 \mathrm{~g}$ of DH (total $68.0 \mathrm{~g}$ ), were mixed and macerated in $680 \mathrm{~mL}$ of deionized water for $30 \mathrm{~min}$, and then extracted twice by refluxing with the same volume of water for $30 \mathrm{~min}$ each. The combined extract was evaporated under a vacuum and freeze-dried to a powder. As shown in Table 1, we calculated the yield of freeze-dried powder of each batch of samples (the yield of freeze-dried powder = the quality of freeze-dried powder obtained / the total quality of crude drug of the Zhi-Zi-Da-Huang decoction) and determined the amount of sample accordingly. A total of $0.2207 \mathrm{~g}$ (equivaled to $1 \mathrm{~g}$ crude drug of the Zhi-Zi-Da-Huang decoction) of freeze-dried powder of ZZDHD was dissolved into $10 \mathrm{~mL} 75 \%$ methanol, followed by ultrasonic extraction for $30 \mathrm{~min}$. The weight lost by the extracted solution was compensated prior to filtration. After centrifuging at $10,000 \mathrm{rpm}$ for $5 \mathrm{~min}$, the supernatant was filtered using a $0.22 \mu \mathrm{m}$ membrane and stored in a refrigerator at $4{ }^{\circ} \mathrm{C}$ for further analysis.

\subsubsection{Preparation of the single-boiled Zhi-Zi-Da-Huang decoction solutions (SB)}

Separately, $30.0 \mathrm{~g}$ of ZZ, ZS, DDC, and DH was weighed, macerated and extracted twice by refluxing with $300 \mathrm{~mL}$ of deionized water for $30 \mathrm{~min}$. After concentrating the samples, the extracts were freeze-dried into powder from water extracts, and the amount of sample was determined according to the yield 
(Table 1). In total, $0.2207 \mathrm{~g}$ (equivalent to $1.0 \mathrm{~g}$ crude drug of the Zhi-Zi-Da-Huang decoction) freeze-dried powder with ZZ, ZS, DDC, and DH in the ratio of (9:12:10:3, W/W/W/W) was weighed and prepared following the method described above.

Table 1

Yield of freeze-dried powder of co-boiled (CB) and single-boiled (SB) Zhi-Zi-DaHuang decoction

\begin{tabular}{|c|c|c|c|c|c|c|}
\hline \multirow[t]{2}{*}{ Sample } & \multirow[t]{2}{*}{ Herbs } & \multicolumn{3}{|c|}{ Freeze-dried Powder/g } & \multirow[t]{2}{*}{ Yield/\% } & \multirow[t]{2}{*}{$\mathrm{RSD} / \%$} \\
\hline & & 1 & 2 & 3 & & \\
\hline $\mathrm{CB}$ & $\mathrm{ZZ}, \mathrm{DH}, \mathrm{ZS}, \mathrm{DDC}$ & 15.01 & 15.69 & 15.11 & $22.44 \pm 0.52$ & 2.32 \\
\hline \multirow[t]{4}{*}{ SB } & $\mathrm{ZZ}$ & 8.58 & 8.64 & 8.42 & $28.46 \pm 0.37$ & 1.31 \\
\hline & $\mathrm{DH}$ & 8.54 & 10.45 & 9.74 & $31.87 \pm 3.25$ & 10.08 \\
\hline & ZS & 5.4 & 6.21 & 6.37 & $19.93 \pm 1.72$ & 8.68 \\
\hline & DDC & 4.71 & 5.58 & 5.27 & $17.28 \pm 1.47$ & 8.5 \\
\hline
\end{tabular}

\subsection{Qualitative analysis with UPLC-qTOF-MS/MS}

\subsubsection{Liquid chromatography}

UPLC was performed with a Waters Acquity Ultra-High-Performance Liquid Chromatography System, equipped with a DAD detector, a quaternary pump and an autosampler. Chromatographic separation was performed on an Acquity UPLC BEH column $(2.1 \times 100 \mathrm{~mm}$ i.d., $1.7 \mu \mathrm{m})$. The mobile phase was composed of solvent $A$ (acetonitrile) and solvent $B(0.5 \%$ formic acid aqueous solution) with a gradient elution program: $5 \%$ A (0-2 min), 5-28\% A (2-5 min), 28\% A (5$11 \mathrm{~min}), 28-92 \% \mathrm{~A}(11-13 \mathrm{~min}), 92-100 \% \mathrm{~A}(13-16 \mathrm{~min}), 100 \% \mathrm{~A}(16-19 \mathrm{~min})$. The flow rate was set at $0.3 \mathrm{~mL} / \mathrm{min}$ with the column temperature maintained at $35^{\circ} \mathrm{C}$ and the sample volume was $1 \mu \mathrm{L}$. The re-equilibration duration was set at 10 min between individual runs.

\subsubsection{Mass spectrometry}

High-accuracy mass spectrometric data were recorded on a Waters Xevo G2-XS Q-Tof mass spectrometer (AB SCIEX, Foster City, CA, USA). Mass spectrometry conditions for MS/MS were set as follows: MS ${ }^{e}$ acquisition mode; capillary voltage, $3.0 \mathrm{kV}$; cone hole voltage, $10 \mathrm{~V}$; cone hole gas flow rate, $50 \mathrm{~L} / \mathrm{h}$; ion source compensation voltage, $80 \mathrm{~V}$; ion source temperature, $150{ }^{\circ} \mathrm{C}$; dissolvent gas temperature, $450^{\circ} \mathrm{C}$. A complete scan was performed in the ESI positive and negative ion mode within the $\mathrm{m} / \mathrm{z} 50-1,200 \mathrm{Da}$ with a cumulative time of $200 \mathrm{~ms}$. The collision voltage is $10-50 \mathrm{~V}$ under positive ion scanning and the collision voltage is 5-60 V under negative ion scanning. Data acquisition and processing were carried out using the Masslynx 4.1 software.

\subsection{Determination of 15 compounds in ZZDHD}

\subsubsection{UPLC-DAD conditions}

The UPLC-DAD was carried out on a Shimadzu LC-30A HPLC system, consisting of a Shimadzu LC-30AT dual pump, a Shimadzu SIL-30A autosampler, a CTO$30 \mathrm{~A}$ column compartment, and an SPD-M30A photodiode array detector. The quantitative samples were detected at $254 \mathrm{~nm}, 283 \mathrm{~nm}$, and $440 \mathrm{~nm}$. Other chromatographic conditions, such as the chromatographic column, mobile phase composition, gradient program, flow rate, column temperature, and injection volume, were the same as those mentioned in Sect. 2.3.1. Each sample was measured in parallel three times.

\subsubsection{Validation of the quantification method}

The system precision was determined by examining six replicate injections of the same sample solution within a day and performing the procedure on three consecutive days. Six independently prepared solutions from the same sample were determined to check the repeatability. The samples were measured at 0,2 , $4,8,12$, and $24 \mathrm{~h}$ to verify for stability. The recovery test was conducted to evaluate the accuracy of this method. The CB sample (0.1103 g, which equated to $0.5 \mathrm{~g}$ crude ZZDHD) was weighed independently six times and spiked with a known amount (approximately equivalent to $0.8,1.0$, and 1.2 times the amount that the actual sample contained) of the corresponding standard compounds. Then, the spiked samples were extracted and quantified with the methods mentioned above.

\subsection{Fingerprint of single-boiled and co-boiled ZZDHD}

\subsubsection{UPLC-DAD conditions}

The qualitative samples were detected at $254 \mathrm{~nm}, 280 \mathrm{~nm}$, and $440 \mathrm{~nm}$ as described in section '2.4.1'.

\subsubsection{Validation of the fingerprint method}

Precision, repeatability, and stability were determined as described in section '2.4.2'.

\subsubsection{Similarity analysis of UPLC fingerprints}

To establish the representative chromatographic fingerprint, a total of 12 samples (six batches of CB (S1-S6), six batches of SB (S7-S12)) were analyzed following the abovementioned methods. The chromatography fingerprint was established using the 'Similarity Evaluation System of Traditional Chinese Medicine Chromatographic Fingerprint' software (China Committee of Pharmacopeia, 2012). 


\subsection{Statistical analysis}

The qTOF-MS/MS data of 12 samples from two methods of ZZDHD preparation were processed using Masslynx 4.1 software. HCA, PCA, and PLS-DA were implemented to characterize the dissimilarities in chemical components among different samples with regard to the processing method. These were operated by SIMCA-P software 14.0 (Umetrics, Umeå, Sweden). Nonparametric tests were used to evaluate the significant differences between the different groups by GraphPad Prism software (GraphPad Prism Software Inc., CA, USA). P $<0.05$ was considered to be significant.

\section{Results}

\subsection{UPLC-qTOF-MS/MS qualitative analysis of chemical constituents in ZZDHD}

The MS spectra were obtained in both positive and negative ion modes. Total ion chromatograms of samples were obtained using the optimized UPLC-qTOFMS/MS system (Fig. 2). In the full scan mass spectra, most authentic compounds exhibited [M-H]- in negative mode or [M+ $\mathrm{H}]+$ in positive mode. The chemical formula of an unknown structure was deduced based on the high accuracy [M-H]-/[M+HCOO]- (in the negative ion mode) or [M + $\mathrm{H}]+/[\mathrm{M}+\mathrm{Na}]+$ (in the positive ion mode) precursor ion. Information on the characteristic constituents, such as $t_{R}(m i n)$, formula, observed $\mathrm{m} / \mathrm{z}$ values, mass error (in ppm), and MS/MS product fragment ions, is summarized in Table 2. In total, 147 compounds were identified in negative and positive ion modes, including 77 known compounds and 70 unknown compounds. Among these compounds, 11, including gallic acid-4-0- $\beta$-D-glucopyranoside, glucosylsyringic acid, Unknown 4, gallate-0-diglucoside, glycerinmonogallete, Unknown 15, 3 ', 4', 5, 5 ', 6, 7-Hexamethoxyflavone, Unknown 59, Unknown 61, Unknown 66, and Unknown 69, were found only in the co-boiled ZZDHD.

Table 2 Characterization compounds of Zhi-Zi-Da-Huang decoction by UPLC-qTOF-MS/MS. 


\begin{tabular}{|c|c|c|c|c|c|c|c|c|c|}
\hline \multirow{2}{*}{$\begin{array}{l}\text { Peak } \\
\text { NO. }\end{array}$} & \multirow[t]{2}{*}{$t_{R} / \min$} & \multirow[t]{2}{*}{ Formula } & \multicolumn{3}{|c|}{ Negative ion $囚 \mathrm{~m} / z$ ) } & \multicolumn{3}{|c|}{ Positive ion $\llbracket m / z)$} & \multirow[t]{2}{*}{ Ider } \\
\hline & & & $\begin{array}{l}\text { Calcd. } \\
\text { mass } \\
(\mathrm{m} / \mathrm{z})\end{array}$ & $\begin{array}{l}\text { Measured } \\
\text { mass } \\
(\mathrm{m} / \mathrm{z})\end{array}$ & $\mathrm{MS} / \mathrm{MS}$ & $\begin{array}{l}\text { Calcd. } \\
\text { mass } \\
(\mathrm{m} / \mathrm{z})\end{array}$ & $\begin{array}{l}\text { Measured } \\
\text { mass }(\mathrm{m} / \mathrm{z})\end{array}$ & $\mathrm{MS} / \mathrm{MS}$ & \\
\hline 1 & 0.80 & $\mathrm{C} 12 \mathrm{H} 22 \mathrm{O} 11$ & 341.1084 & 341.1088 & 387.1143[M+HCOO]- & & & $\begin{array}{l}365.1028[\mathrm{M}+\mathrm{Na}]+\rrbracket \\
707.1824[2 \mathrm{M}+\mathrm{Na}]+\end{array}$ & Unk \\
\hline 2 & 0.83 & C7H12O6 & 191.0497 & 191.0562 & $\begin{array}{l}\text { 127.039[M-H-2H2O- } \\
\text { CO]- } 囚 \\
109.0290[\mathrm{M}-\mathrm{H}-3 \mathrm{H} 2 \mathrm{O}- \\
\text { CO]- } 囚 \\
93.0342[\mathrm{M}-\mathrm{H}-3 \mathrm{H} 2 \mathrm{O}- \\
\text { CO2]- }\end{array}$ & & & $215.0492[\mathrm{M}+\mathrm{Na}]+$ & Quil \\
\hline 3 & 1.04 & $\mathrm{C} 6 \mathrm{H} 807$ & 191.0192 & 191.0195 & & & & $215.0162[\mathrm{M}+\mathrm{Na}]+$ & Citri \\
\hline 4 & 1.05 & $\mathrm{C} 12 \mathrm{H} 1608$ & 287.0767 & 287.0772 & $\begin{array}{l}333.0831[\mathrm{M}+\mathrm{HCOO}]-\rrbracket \\
125.0232[\mathrm{M}-\mathrm{H}-\mathrm{Glc}]-\end{array}$ & & & $\begin{array}{l}311.0742[\mathrm{M}+\mathrm{Na}]+\rrbracket \\
127.0388[\mathrm{M}+\mathrm{H}-\mathrm{Gl}]+\end{array}$ & Unk \\
\hline 5 & 1.16 & $\mathrm{C} 13 \mathrm{H} 16010$ & 331.0665 & 331.0673 & $\begin{array}{l}271.0462[\mathrm{M}-\mathrm{H}- \\
\mathrm{C} 2 \mathrm{H} 402]-\mathrm{\Xi} \\
211.0232[\mathrm{M}-\mathrm{H}- \\
2 \mathrm{C} 2 \mathrm{H} 402]-\mathrm{\Omega} \\
169.0137[\mathrm{M}-\mathrm{H}- \\
2 \mathrm{C} 2 \mathrm{H} 402-\mathrm{C} 2 \mathrm{H} 2 \mathrm{O}]-\end{array}$ & & & $355.0575[\mathrm{M}+\mathrm{Na}]+$ & $\begin{array}{l}\text { Gall } \\
\text { gluc }\end{array}$ \\
\hline 6 & 1.27 & C31H47024 & & & & 803.2457 & 803.2443 & $825.2187[\mathrm{M}+\mathrm{Na}]+$ & Unk \\
\hline 7 & 1.32 & $\mathrm{C} 13 \mathrm{H} 16010$ & 331.0665 & 331.0669 & $\begin{array}{l}\text { 169.0137[M-H-Glc]- } \rrbracket \\
151.0023\left[\mathrm{M}-\mathrm{H}-\mathrm{Glc}^{-}\right. \\
\mathrm{H} 2 \mathrm{O}]-\end{array}$ & & & & Glur \\
\hline 8 & 1.43 & C7H6O5 & 169.0137 & 169.0136 & 125.0239[M-H-CO2]- & 171.0293 & 171.0285 & $127.0387[\mathrm{M}+\mathrm{H}-\mathrm{CO} 2]+$ & Gall \\
\hline 9 & 1.47 & $\mathrm{C} 30 \mathrm{H} 22 \mathrm{O}$ & 493.1287 & 493.1289 & & & & 517.1169[M+Na]+ & Unk \\
\hline 10 & 1.48 & $\mathrm{C} 16 \mathrm{H} 22 \mathrm{O} 11$ & 389.1084 & 389.1091 & 227.0175[M-H-Glc]- & & & $\begin{array}{l}359.0906[\mathrm{M}+\mathrm{Na}]+\mathbb{\square} \\
229.1367[\mathrm{M}+\mathrm{H}-\mathrm{Glc}]+\end{array}$ & $\begin{array}{l}\text { Dea } \\
\text { acic }\end{array}$ \\
\hline 11 & 1.52 & $\mathrm{C} 25 \mathrm{H} 32 \mathrm{O} 16$ & 587.1765 & 587.1773 & & 589.1921 & 589.1773 & $611.1543[\mathrm{M}+\mathrm{Na}]+$ & Unk \\
\hline 12 & 1.52 & $\mathrm{C} 16 \mathrm{H} 20011$ & 387.0927 & 387.0937 & $\begin{array}{l}\text { 775.18451[M+HCOO]- } \square \\
225.0363[\mathrm{M}-\mathrm{H}-\mathrm{GlC}]-\end{array}$ & & & $411.0834[\mathrm{M}+\mathrm{Na}]+$ & Unk \\
\hline 13 & 1.56 & $\mathrm{C} 19 \mathrm{H} 26015$ & 493.1193 & 493.1200 & $\begin{array}{l}\text { 169.0132[M-H-2Glc]- } \\
\text { 125.0233[M-H-2Glc- } \\
\text { CO2]- }\end{array}$ & & & $\begin{array}{l}517.1115[\mathrm{M}+\mathrm{Na}]+\rrbracket \\
127.0354[\mathrm{M}+\mathrm{H}-2 \mathrm{Glc}-\mathrm{CO} 2]+\end{array}$ & Gall \\
\hline 14 & 1.66 & $\mathrm{C} 14 \mathrm{H} 16011$ & 359.0614 & 359.0616 & $\begin{array}{l}\text { 315.0681[M-H-CO2]- } \\
153.0173[\mathrm{M}-\mathrm{H}-\mathrm{CO} 2- \\
\text { Glc]- } \\
125.0232[\mathrm{M}-\mathrm{H}-\mathrm{CO} 2- \\
\text { Glc-CO]- }\end{array}$ & & & $\begin{array}{l}383.0541[\mathrm{M}+\mathrm{Na}]+\rrbracket \\
127.0378[\mathrm{M}+\mathrm{H}-\mathrm{CO} 2-\mathrm{Glc}- \\
\mathrm{CO}]+\end{array}$ & Unk \\
\hline 15 & 1.76 & $\mathrm{C} 16 \mathrm{H} 22 \mathrm{O} 12$ & 405.1033 & 405.1039 & $\begin{array}{l}287.0750[\mathrm{M}-\mathrm{H}- \\
\text { C8H8O]-囚 } \\
\text { 125.0232[M-H-C8H8O- } \\
\text { Glc]- }\end{array}$ & & & $\begin{array}{l}429.0963[\mathrm{M}+\mathrm{Na}]+\rrbracket \\
245.09784[\mathrm{M}+\mathrm{H}-\mathrm{Gl}]+\end{array}$ & Unk \\
\hline 16 & 1.84 & $\mathrm{C} 16 \mathrm{H} 22 \mathrm{O} 10$ & 373.1135 & 373.1190 & $\begin{array}{l}211.0629[\mathrm{M}-\mathrm{H}-\mathrm{Glu}]-囚 \\
193.0534[\mathrm{M}-\mathrm{H}-\mathrm{Glu}- \\
\text { H2O]- }\end{array}$ & & & $\begin{array}{l}\text { 397.1061[M+Na]+凹 } \\
229.1398[\mathrm{M}+\mathrm{H}-\mathrm{C} 8 \mathrm{H} 802]+\end{array}$ & Gar \\
\hline 17 & 1.89 & $\mathrm{C} 16 \mathrm{H} 22 \mathrm{O} 12$ & 405.1033 & 405.1039 & $\begin{array}{l}287.0716[\mathrm{M}-\mathrm{H}- \\
\mathrm{C} 8 \mathrm{H} 80] \rrbracket \\
125.0232[\mathrm{M}-\mathrm{H}-\mathrm{C} 8 \mathrm{H} 80- \\
\text { Glc]- }\end{array}$ & & & 429.0963[M+Na]+ & Unk \\
\hline 18 & 1.97 & $\mathrm{C} 16 \mathrm{H} 24011$ & 391.1240 & 391.1241 & $\begin{array}{l}229.0721[\mathrm{M}-\mathrm{H}-\mathrm{Glc}]-\rrbracket \\
211.0659[\mathrm{M}-\mathrm{H}-\mathrm{Glc}- \\
\mathrm{H} 20]-\mathrm{W} \\
167.0694[\mathrm{M}-\mathrm{H}-\mathrm{Glc}- \\
\text { H2O-CO2]- }\end{array}$ & & & $415.1135[\mathrm{M}+\mathrm{Na}]+$ & Car! \\
\hline 19 & 2.11 & $\mathrm{C} 10 \mathrm{H} 1207$ & 243.0505 & 243.0502 & $\begin{array}{l}\text { 169.0124[M-H- } \\
\text { C3H6O2]区 } \\
125.0237[\mathrm{M}-\mathrm{H}- \\
\text { C3H6O2-CO2] }\end{array}$ & & & & Glyc \\
\hline 20 & 2.36 & $\mathrm{C} 16 \mathrm{H} 2609$ & 361.1499 & 361.1499 & 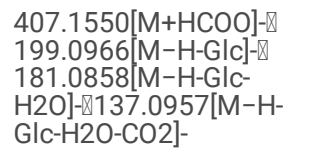 & & & $\begin{array}{l}385.1436[\mathrm{M}+\mathrm{Na}]+\square \\
201.0429[\mathrm{M}+\mathrm{H}-\mathrm{Gl} \mathrm{c}]+\square\end{array}$ & Unk \\
\hline 21 & 2.43 & $\mathrm{C} 16 \mathrm{H} 24011$ & 391.1240 & 391.1240 & $783.2554[2 M-H]-\otimes$ & & & $415.1177[\mathrm{M}+\mathrm{Na}]+\rrbracket$ & Sha \\
\hline
\end{tabular}




\begin{tabular}{|c|c|c|c|c|c|}
\hline 22 & 2.52 & $\mathrm{C} 16 \mathrm{H} 2609$ & 361.1499 & 361.1497 & 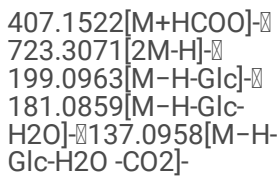 \\
\hline
\end{tabular}

385.1395[M+Na]+\ Unk

$23 \quad 2.58 \quad \mathrm{C} 11 \mathrm{H} 1207 \quad 255.0505 \quad 255.0505 \quad 211.0600[\mathrm{M}-\mathrm{H}-\mathrm{CO} 2]-[$

93.0343[M-H-Glc]-

$\begin{array}{llllll}24 & 2.84 & \mathrm{C} 16 \mathrm{H} 2409 & 359.1342 & 359.1346 & 405.1399[\mathrm{M}+\mathrm{HCOO}]-\mathbb{8} \\ & & & & 197.9606[\mathrm{M}-\mathrm{H}-\mathrm{Glc}]-\end{array}$

$279.0455[\mathrm{M}+\mathrm{Na}]+\square$
$229.1398[\mathrm{M}+\mathrm{H}-\mathrm{CO}]+$

Unk

$25 \quad 3.23 \quad \mathrm{C} 17 \mathrm{H} 24011 \quad 403.1240 \quad 403.1246 \quad 449.1295[\mathrm{M}+\mathrm{HCOO}]-\mathbb{\nabla}$

241.0705[M-H-Glc]-

$743.2716[2 \mathrm{M}+\mathrm{Na}]+$

$427.1147[\mathrm{M}+\mathrm{Na}]+\rrbracket$

$831.2433[2 \mathrm{M}+\mathrm{Na}]+\mathbb{Q}$

$265.0281[\mathrm{M}+\mathrm{Na}-\mathrm{Gl} \mathrm{c}]+\rrbracket$

$\begin{array}{lllll}26 & 3.44 & \mathrm{C} 13 \mathrm{H} 1409 & 313.0560 & 313.0558\end{array}$

$337.0496[\mathrm{M}+\mathrm{Na}]+\rrbracket$

$651.1091[2 \mathrm{M}+\mathrm{Na}]+\square$

$319.0424[\mathrm{M}+\mathrm{Na}-\mathrm{H} 2 \mathrm{O}]+\square$

$301.0753[\mathrm{M}+\mathrm{Na}-2 \mathrm{H} 2 \mathrm{O}]+\square$

\begin{tabular}{|c|c|c|c|c|c|}
\hline \multirow[t]{2}{*}{27} & 3.44 & $\mathrm{C} 16 \mathrm{H} 1809$ & 353.0873 & 353.0869 & $\begin{array}{l}\text { 179.0336[M-H-- } \\
\mathrm{C} 7 \mathrm{H} 1005]-\triangle\end{array}$ \\
\hline & & & & & $\begin{array}{l}\text { 135.0437[M-H- } \\
\text { C7H1005-CO2] }\end{array}$ \\
\hline
\end{tabular}

$377.0836[\mathrm{M}+\mathrm{Na}]+$

Chls

\begin{tabular}{|c|c|c|c|c|}
\hline $28 \quad 3.52$ & $\mathrm{C} 16 \mathrm{H} 22011$ & 389.1084 & 389.1074 & $\begin{array}{l}345.1171[\mathrm{M}-\mathrm{H}-\mathrm{CO} 2]-\mathrm{Z} \\
209.0306[\mathrm{M}-\mathrm{H}-\mathrm{GlC}- \\
\mathrm{H} 2 \mathrm{O}]-\otimes 191.0183[\mathrm{M}-\mathrm{H} \\
\text { Glc-2H2O]- }\end{array}$ \\
\hline
\end{tabular}

413.1022[M+Na]+ Mor

\begin{tabular}{|c|c|c|c|c|c|}
\hline 29 & 3.59 & $\mathrm{C} 27 \mathrm{H} 22$ & 345.1643 & 345.1616 & \\
\hline 30 & 3.66 & $\mathrm{C} 17 \mathrm{H} 24011$ & 403.1240 & 403.1242 & $\begin{array}{l}449.1311[\mathrm{M}+\mathrm{HCOO}]- \\
241.0710[\mathrm{M}-\mathrm{H}-\mathrm{Glc}]-\mathrm{Q} \\
223.0605[[\mathrm{M}-\mathrm{H}-\mathrm{Gl}- \\
\mathrm{H} 2 \mathrm{O}]-\end{array}$ \\
\hline
\end{tabular}

\begin{tabular}{|c|c|c|c|c|c|}
\hline 31 & 3.69 & $\mathrm{C} 16 \mathrm{H} 2608$ & 345.1549 & 345.1554 & $\begin{array}{l}391.1607[\mathrm{M}+\mathrm{HCOO}]-\mathbb{} \\
691.3181[2 \mathrm{M}-\mathrm{H}]-\mathbb{} \\
165.0913[\mathrm{M}-\mathrm{H}-\mathrm{GlC}- \\
\text { H2O]- }\end{array}$ \\
\hline 32 & 3.78 & $\mathrm{C} 17 \mathrm{H} 26011$ & 405.1397 & 405.1397 & $451.1242[\mathrm{M}+\mathrm{HCOO}]-$ \\
\hline 33 & 3.83 & C17 H24 011 & 403.1240 & 403.1238 & $\begin{array}{l}449.1300[\mathrm{M}+\mathrm{HCOO}]-\mathbb{} \\
241.0700[\mathrm{M}-\mathrm{H}-\mathrm{GlC}]-\end{array}$ \\
\hline
\end{tabular}

369.1491[M+Na]+\- Unk 715.1443[2M+Na]+

$427.1232[\mathrm{M}+\mathrm{Na}]+\rrbracket$

$831.2433[2 \mathrm{M}+\mathrm{Na}]+\square$ 409.1067[M+Na-H2O]+ฉ

$265.0664[\mathrm{M}+\mathrm{Na}-\mathrm{Glc}]+\mathbb{Z}$ 247.0527[M+Na-Glc-H2O]+

$369.1530[\mathrm{M}+\mathrm{Na}]+\rrbracket$

207.0651[M+Na-Glc]+区

189.0544[M+Na-Glc-H2O]+

427.1147[M+Na]+

$427.1190[\mathrm{M}+\mathrm{Na}]+\mathbb{Q}$

$831.2433[2 \mathrm{M}+\mathrm{Na}]+\mathbb{Q}$

$265.0686[\mathrm{M}+\mathrm{Na}-\mathrm{Gl}]+\rrbracket$

247.0582[M+Na-Glc-H2O]+

\begin{tabular}{|c|c|c|c|c|c|c|c|c|}
\hline 34 & 3.98 & $\mathrm{C} 21 \mathrm{H} 34012$ & 477.1972 & 477.1975 & 523.1696[M+HCOO]- & & & \\
\hline 35 & 4.01 & $\mathrm{C} 16 \mathrm{H} 2608$ & 345.1549 & 345.1553 & 391.1608[M+HCOO]- & 347.1706 & 347.1700 & 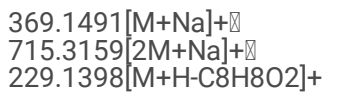 \\
\hline 36 & 4.06 & $\mathrm{C} 13 \mathrm{H} 1409$ & 313.0560 & 313.0562 & $627.1202[2 \mathrm{M}-\mathrm{H}]-$ & & & $\begin{array}{l}337.0496[\mathrm{M}+\mathrm{Na}]+\rrbracket \\
651.1177[2 \mathrm{M}+\mathrm{Na}]+\end{array}$ \\
\hline 37 & 4.13 & $\mathrm{C} 17 \mathrm{H} 28010$ & 391.1604 & 391.1604 & & & & \\
\hline 38 & 4.16 & $\mathrm{C} 15 \mathrm{H} 1406$ & 289.0716 & 289.0708 & $245.0807[\mathrm{M}-\mathrm{H}-\mathrm{CO} 2]-$ & 291.0869 & 291.0863 & \\
\hline 39 & 4.19 & $\mathrm{C} 16 \mathrm{H} 1809$ & 353.0873 & 353.0874 & $\begin{array}{l}\text { 707.1819[M+HCOO]- } \mathrm{X} \\
\text { 191.0522[M-H-Glc] }\end{array}$ & 355.1029 & 355.1022 & $\begin{array}{l}377.0781[\mathrm{M}+\mathrm{Na}]+\rrbracket \\
731.1740[2 \mathrm{M}+\mathrm{Na}]+\end{array}$ \\
\hline 40 & 4.23 & $\mathrm{C} 13 \mathrm{H} 1409$ & 313.0560 & 313.0578 & $627.1203[2 \mathrm{M}-\mathrm{H}]-$ & & & $337.0496[\mathrm{M}+\mathrm{Na}]+$ \\
\hline 41 & 4.33 & $\mathrm{C} 23 \mathrm{H} 34015$ & 549.1819 & 549.1922 & 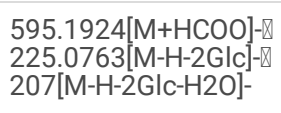 & & & $\begin{array}{l}573.1796[\mathrm{M}+\mathrm{Na}]+\bigotimes \\
1123.3578[2 \mathrm{M}+\mathrm{Na}]+\end{array}$ \\
\hline 42 & 4.39 & $\mathrm{C} 11 \mathrm{H} 12 \mathrm{O} 6$ & 239.0556 & 239.0562 & $\begin{array}{l}\text { 195.0656[M-H-CO2]-区 } \\
177.0546[[\mathrm{M}-\mathrm{H}-\mathrm{CO} 2- \\
\mathrm{H} 2 \mathrm{O}]-\otimes 149.0589[\mathrm{M}-\mathrm{H}- \\
\mathrm{CO} 2-\mathrm{H} 2 \mathrm{C}-\mathrm{CO}]-\end{array}$ & & & 263.0513[M+Na]+ \\
\hline 43 & 4.48 & $\mathrm{C} 21 \mathrm{H} 26013$ & 485.1295 & 485.1306 & $\begin{array}{l}531.1357[\mathrm{M}+\mathrm{HCOO}]-\square \\
971.2648[2 \mathrm{M}-\mathrm{H}]-\square\end{array}$ & 487.1452 & 487.1457 & 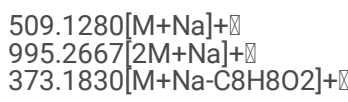 \\
\hline
\end{tabular}

Page 7/28 


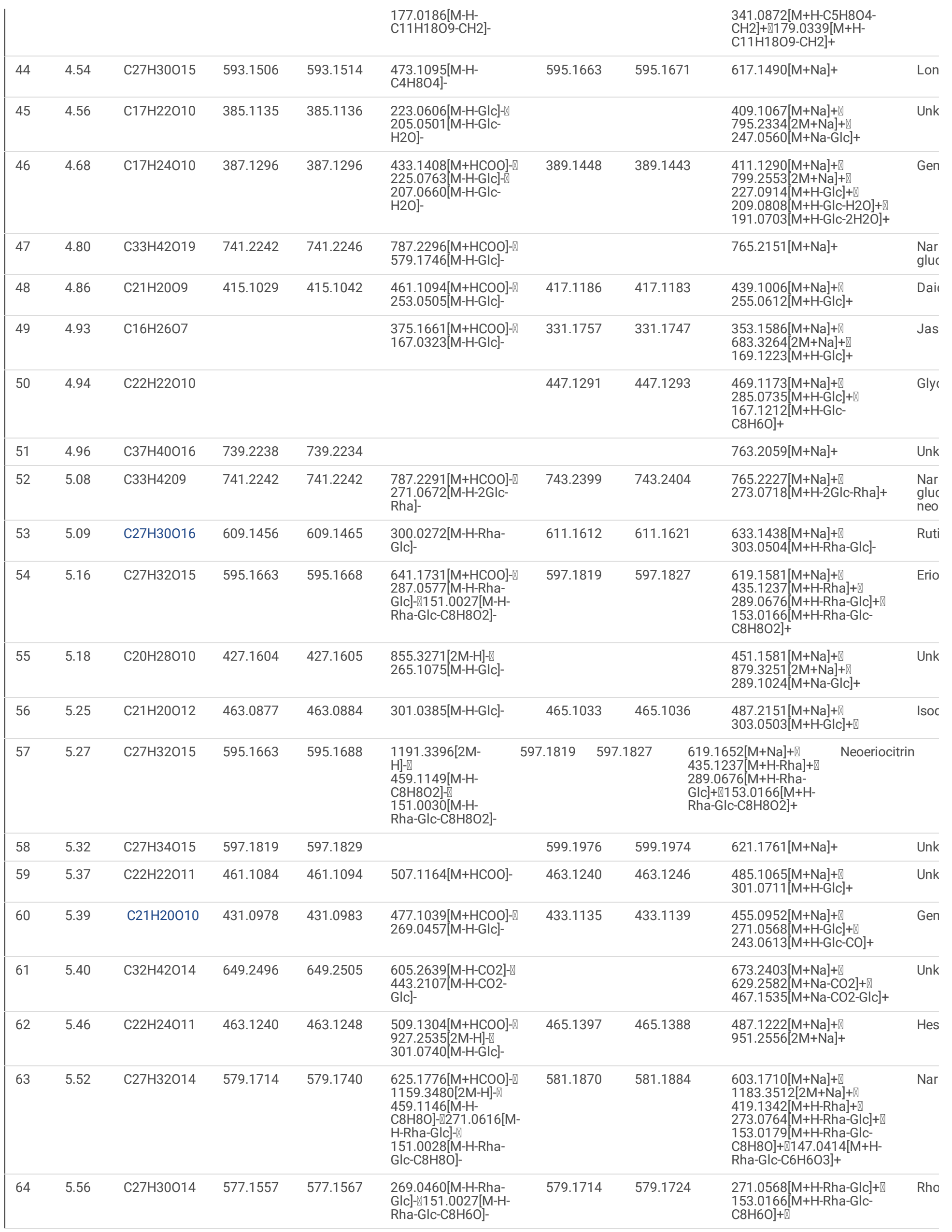




\begin{tabular}{|c|c|c|c|c|c|c|c|c|c|}
\hline 65 & 5.58 & $\mathrm{C} 44 \mathrm{H} 64 \mathrm{O} 24$ & 975.3709 & 975.3696 & $\begin{array}{l}\text { 1021.3752[M+HCOO]- } ₫ \\
651.2679[\mathrm{M}-\mathrm{H}-2 \mathrm{Glc}]-\rrbracket \\
327.1615[\mathrm{M}-\mathrm{H}-2 \mathrm{Glc}- \\
\text { 2Glc]- } ₫ 283.1700[\mathrm{M}-\mathrm{H}- \\
\text { 2Glc-2Glc-CO2]- } \\
-\end{array}$ & & & 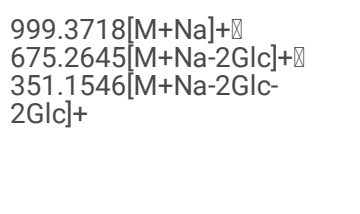 & Cror \\
\hline 66 & 5.62 & C27H32O14 & 579.1714 & 579.1788 & 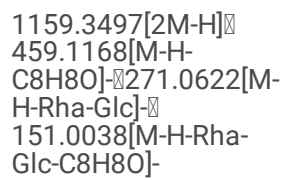 & 581.1870 & 581.1890 & $\begin{array}{l}\text { 603.1709[M+Na]+区 } \\
435.1293[\mathrm{M}+\mathrm{H}-\mathrm{Rha}]+\rrbracket \\
273.0752[\mathrm{M}+\mathrm{H}-\mathrm{Rha}-\mathrm{Glc}]+\rrbracket \\
\text { 153.0166[M+H-Rha-Glc- } \\
\text { C8H80]+ }\end{array}$ & Nar \\
\hline 67 & 5.73 & $\mathrm{C} 32 \mathrm{H} 40017$ & 695.2222 & 695.2214 & $741.2259[\mathrm{M}+\mathrm{HCOO}]-$ & 697.2344 & 697.2365 & $719.222[\mathrm{M}+\mathrm{Na}]+$ & Unk \\
\hline 68 & 5.73 & $\mathrm{C} 28 \mathrm{H} 34015$ & 609.1819 & 609.1841 & $\begin{array}{l}\text { 301.0722[M-H-Rha- } \\
\text { Glc]-囚286.0485[M-H- } \\
\text { Rha-Glc-CH3]- }\end{array}$ & 611.1976 & 611.1992 & 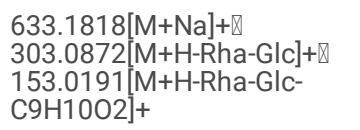 & Hes \\
\hline 69 & 5.81 & С31Н32O16 & 659.1612 & 659.1771 & 497.1339[M-H-Glc]- & 661.1769 & 661.1788 & $683.1613[\mathrm{M}+\mathrm{Na}]+$ & $\begin{array}{l}3 \otimes 4 \\
\text { hyd } \\
\text { glut }\end{array}$ \\
\hline 70 & 5.91 & С38H54019 & 813.3181 & 813.3193 & 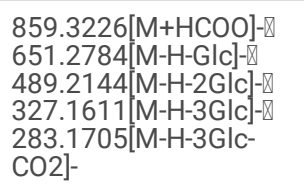 & & & 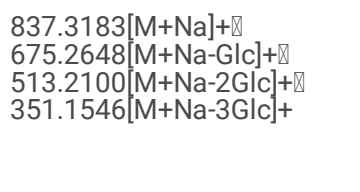 & Cror \\
\hline 71 & 5.92 & $\mathrm{C} 15 \mathrm{H} 1005$ & 269.0450 & 269.0454 & $\begin{array}{l}\text { 151.0027[M-H- } \\
\text { C8H6O]- }\end{array}$ & 271.0606 & 271.0606 & 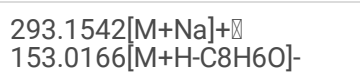 & Apic \\
\hline 72 & 5.98 & $\mathrm{C} 22 \mathrm{H} 24011$ & 463.1240 & 463.1254 & 301.0740[M-H-Glc]- & 465.1430 & 465.1430 & $\begin{array}{l}\text { 487.1227[M+Na]+区 } \\
303.0869[\mathrm{M}+\mathrm{H}-\mathrm{Glc}]+\end{array}$ & $\begin{array}{l}\text { Hes } \\
\text { isor }\end{array}$ \\
\hline 73 & 5.98 & $\mathrm{C} 27 \mathrm{H} 36012$ & 551.2129 & 551.2136 & $\begin{array}{l}\text { 597.2201[M+HCOO]- } \mathrm{M} \\
1103.4235[2 \mathrm{M}-\mathrm{H}]-\end{array}$ & & & $575.2907[\mathrm{M}+\mathrm{Na}]+$ & Unk \\
\hline 74 & 6.02 & $\mathrm{C} 27 \mathrm{H} 36012$ & 551.2129 & 551.2150 & $\begin{array}{l}597.2205[\mathrm{M}+\mathrm{HCOO}]-\mathbb{} \\
1103.4350[2 \mathrm{M}-\mathrm{H}]-\end{array}$ & 553.2285 & 553.2296 & $\begin{array}{l}575.2097[\mathrm{M}+\mathrm{Na}]+\rrbracket \\
1127.4340[2 \mathrm{M}+\mathrm{Na}]+\rrbracket \\
207.0644[\mathrm{M}+\mathrm{H}-2 \mathrm{Glc}-\mathrm{CO}]+\end{array}$ & Unk \\
\hline 75 & 6.08 & $\mathrm{C} 27 \mathrm{H} 28013$ & 559.1452 & 559.1465 & $\begin{array}{l}\text { 397.1143[M-H- } \\
\text { C9H703]-囚 } \\
\text { 223.0609[M-H- } \\
\text { C9H703-Rha-CO]- }\end{array}$ & 561.1633 & 561.1633 & $\begin{array}{l}583.1441[\mathrm{M}+\mathrm{Na}]+\rrbracket \\
1143.2992[2 \mathrm{M}+\mathrm{Na}]+\end{array}$ & $\begin{array}{l}\text { 4-Si } \\
\text { caft }\end{array}$ \\
\hline 76 & 6.08 & $\mathrm{C} 22 \mathrm{H} 22 \mathrm{O} 11$ & 461.1084 & 461.1095 & 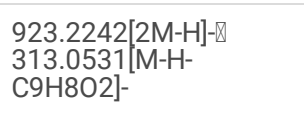 & & & $\begin{array}{l}\text { 485.1050[M+Na]+囚 } \\
315.0816[\mathrm{M}+\mathrm{H}-\mathrm{C} 9 \mathrm{H} 802]+\end{array}$ & $\begin{array}{l}\text { 2-O- } \\
\text { gluc }\end{array}$ \\
\hline 77 & 6.17 & $\mathrm{C} 28 \mathrm{H} 34014$ & 593.1870 & 593.1887 & $\begin{array}{l}\text { 639.1938[M+HCOO]- } \\
\text { 285.0767[M-H-Rha- } \\
\text { Glc]- }\end{array}$ & 595.2027 & 595.2145 & $617.1866[\mathrm{M}+\mathrm{Na}]+$ & $\begin{array}{l}61-0 \\
\text { Sin }\end{array}$ \\
\hline 78 & 6.23 & $\mathrm{C} 22 \mathrm{H} 36013$ & 461.1143 & 461.2225 & 507.218[M+HCOO]- & & & $\begin{array}{l}\text { 485.1997[M+Na]+区 } \\
301.1031[\mathrm{M}+\mathrm{H}-\mathrm{Gl}]+\end{array}$ & Unk \\
\hline 79 & 6.32 & $\mathrm{C} 22 \mathrm{H} 22 \mathrm{O} 11$ & 461.1084 & 461.1092 & 299.0593[M-H-Glc]- & & & $\begin{array}{l}\text { 485.1031[M+Na]+区 } \\
301.1031[\mathrm{M}+\mathrm{H}-\mathrm{Glc}]+\end{array}$ & $\begin{array}{l}1-0- \\
\text { cinr } \\
\text { gluc }\end{array}$ \\
\hline 80 & 6.35 & $\mathrm{C} 55 \mathrm{H} 74 \mathrm{O} 28$ & 1181.4228 & 1181.4275 & $\begin{array}{l}\text { 857[M-H-2Glc]- } ₫ \\
651.2679[\mathrm{M}-\mathrm{H}-3 \mathrm{Glc}- \\
\text { CO2]- }\end{array}$ & & & $\begin{array}{l}\text { 881.3163[M+Na-2Glc]- } 8 \\
\text { 675.2595[M+Na-3Glc-CO2]- }\end{array}$ & Unk \\
\hline 81 & 6.36 & $\mathrm{C} 32 \mathrm{H} 42 \mathrm{O} 13$ & 633.2547 & 633.2558 & $\begin{array}{l}\text { 427.2139[M-H-GlC- } \\
\text { CO2]- }\end{array}$ & & & $657.2484[\mathrm{M}+\mathrm{Na}]+$ & Unk \\
\hline 82 & 6.41 & $\mathrm{C} 15 \mathrm{H} 1004$ & 253.0501 & 253.0503 & & 255.0657 & 255.0659 & $277.0447[\mathrm{M}+\mathrm{Na}]+$ & Daiı \\
\hline 83 & 6.60 & $\mathrm{C} 16 \mathrm{H} 1205$ & & & & 285.0763 & 285.0763 & $307.0584[\mathrm{M}+\mathrm{Na}]+$ & Glyc \\
\hline 84 & 6.77 & С38H47010 & & & & 663.3169 & 663.3191 & $685.3001[\mathrm{M}+\mathrm{Na}]+$ & Unk \\
\hline 85 & 6.84 & $\mathrm{C} 11 \mathrm{H} 604$ & 201.0188 & 201.0183 & $\begin{array}{l}\text { 173.0263[M-H-CO]- } \square \\
145.0292[\mathrm{M}-\mathrm{H}-2 \mathrm{CO}]-\mathrm{Q} \\
117.0343[\mathrm{M}-\mathrm{H}-3 \mathrm{CO}]-\end{array}$ & 203.0344 & 203.0324 & & Ber! \\
\hline 86 & 7.00 & $\mathrm{C} 28 \mathrm{H} 34014$ & 593.1870 & 593.1867 & 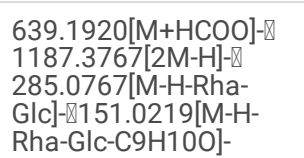 & 595.2027 & 595.1996 & 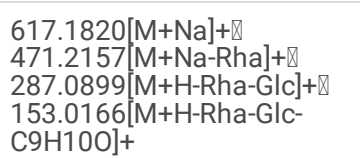 & Pon \\
\hline
\end{tabular}




\begin{tabular}{|c|c|c|c|c|c|c|c|c|c|}
\hline 87 & 7.08 & $\mathrm{C} 21 \mathrm{H} 20010$ & 431.0975 & 431.0975 & $\begin{array}{l}\text { 285.0767[M-H- } \\
\text { C6H1004]- }\end{array}$ & & & $\begin{array}{l}\text { 455.0920[M+Na]+ه } \\
287.0913[\mathrm{M}+\mathrm{H}-\mathrm{C} 6 \mathrm{H} 1004]+\end{array}$ & $\begin{array}{l}\text { Alot } \\
\text { gluc }\end{array}$ \\
\hline 88 & 7.15 & $\mathrm{C} 21 \mathrm{H} 20010$ & 431.0987 & 431.0968 & $\begin{array}{l}269.0444[\mathrm{M}-\mathrm{H}-\mathrm{GlC}]-\mathbb{} \\
225.0544[\mathrm{M}-\mathrm{H}-\mathrm{GlC}- \\
\text { CO2]- }\end{array}$ & & & $\begin{array}{l}\text { 455.0941[M+Na]+囚 } \\
271.9568[\mathrm{M}+\mathrm{H}-\mathrm{Glc}]-\end{array}$ & $\begin{array}{l}\text { Eme } \\
\text { Glui }\end{array}$ \\
\hline 89 & 7.24 & С27H36011 & 535.2179 & 535.2169 & & 537.2330 & 537.2336 & $559.2155[\mathrm{M}+\mathrm{Na}]+$ & $\begin{array}{l}61-0 \\
\text { jasr }\end{array}$ \\
\hline 90 & 7.35 & $\mathrm{C} 42 \mathrm{H} 4507$ & 661.3346 & 661.3346 & & 663.3169 & 663.3190 & 355.0999[M+H-Rha-Glc]+ & Unk \\
\hline 91 & 7.60 & $\mathrm{C} 20 \mathrm{H} 18010$ & 417.0822 & 417.0811 & & & & $441.0772[\mathrm{M}+\mathrm{Na}]+$ & Unk \\
\hline 92 & 7.65 & $\mathrm{C} 28 \mathrm{H} 34014$ & 593.1870 & 593.1860 & 431.1326[M-H-Glc]- & & & & $\begin{array}{l}6 \\
\text { gen }\end{array}$ \\
\hline 93 & 7.91 & $\mathrm{C} 15 \mathrm{H} 1005$ & 269.0450 & 269.0443 & & 271.0606 & 271.0597 & $541.0952[2 \mathrm{M}+\mathrm{H}]+$ & Gen \\
\hline 94 & 7.97 & $\mathrm{C} 15 \mathrm{H} 1205$ & 271.0599 & 271.0606 & $\begin{array}{l}\text { 151.0024[M-H- } \\
\text { C8H8O]- } 107.0127[\mathrm{M}- \\
\mathrm{H}-\mathrm{C} 8 \mathrm{H} 80-\mathrm{CO} 2]-\end{array}$ & 273.0763 & 273.0755 & 153.0166[M-H-C8H8O]+ & Nar \\
\hline 95 & 8.32 & C48H59O22 & 987.3498 & 987.3456 & $\begin{array}{l}\text { 825.3241[M-H-Glc]- } \rrbracket \\
\text { 663.2418[M-H-2Glc]- } ₫ \\
\text { 501.2146[M-H-3Glc]- }\end{array}$ & & & 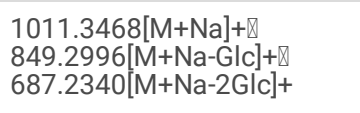 & Unk \\
\hline 96 & 8.70 & $\mathrm{C} 15 \mathrm{H} 1604$ & & & & 261.1127 & 261.1117 & $\begin{array}{l}283.0938[\mathrm{M}+\mathrm{Na}]+\rrbracket \\
243.1011[\mathrm{M}+\mathrm{H}-\mathrm{H} 2 \mathrm{O}]+\end{array}$ & Mer \\
\hline 97 & 8.71 & $\mathrm{C} 21 \mathrm{H} 2406$ & 371.1495 & 371.1490 & 743.3033[2M-H]- & & & $395.1471[\mathrm{M}+\mathrm{Na}]+$ & Unk \\
\hline 98 & 8.85 & $\mathrm{C} 32 \mathrm{H} 44014$ & 651.2653 & 651.2648 & 697.2690[M+HCOO]- $ه$ & & & & Unk \\
\hline 99 & 9.01 & $\mathrm{C} 32 \mathrm{H} 44014$ & 651.2653 & 651.2650 & $\begin{array}{l}\text { 697.2705[M+HCOO]- } \mathrm{M} \\
327.1615[\mathrm{M}-\mathrm{H}-\mathrm{Glc}]-\square \\
283.1715[\mathrm{M}-\mathrm{H}-\mathrm{Gl}- \\
\text { CO2]- } \otimes 239.1821[\mathrm{M}-\mathrm{H}- \\
\text { Glc-2CO2]- }\end{array}$ & & & $\begin{array}{l}\text { 675.2297[M+Na]+凹 } \\
351.1546[\mathrm{M}+\mathrm{Na}-2 \mathrm{Glc}]+\end{array}$ & Cror \\
\hline 100 & 9.29 & $\mathrm{C} 21 \mathrm{H} 2406$ & 371.1494 & 371.1495 & 743.3068[2M-H]- & & & $395.1430[\mathrm{M}+\mathrm{Na}]+$ & Unk \\
\hline 101 & 9.50 & $\mathrm{C} 44 \mathrm{H} 64 \mathrm{O} 24$ & 975.3709 & 975.3678 & $\begin{array}{l}\text { 1021.3727[M+HCOO]-- } \\
651.2679[\mathrm{M}-\mathrm{H}-2 \mathrm{Glc}]-囚 \\
327.1615[\mathrm{M}-\mathrm{H}-2 \mathrm{Glc}- \\
\text { 2Glc]- } 283.1715[\mathrm{M}-\mathrm{H}- \\
\text { 2Glc-2Glc-CO2]- }\end{array}$ & & & 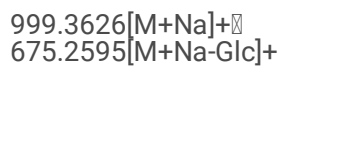 & Cror \\
\hline 102 & 10.16 & $\mathrm{C} 21 \mathrm{H} 22 \mathrm{O} 5$ & & & & 355.1545 & 355.1528 & $\begin{array}{l}\text { 203.0178[M+H-C10H16O]+凹 } \\
147.0430[\mathrm{M}+\mathrm{H}-\mathrm{C} 10 \mathrm{H} 16 \mathrm{O}- \\
2 \mathrm{CO}]+\end{array}$ & Isor \\
\hline 103 & 10.23 & $\mathrm{C} 2 \mathrm{OH} 1809$ & 401.0873 & 401.0868 & $\begin{array}{l}\text { 357.0953[M-H-CO2]- } / \\
313.0723[\mathrm{M}-\mathrm{H}-2 \mathrm{CO} 2]-\end{array}$ & & & & Unk \\
\hline 104 & 11.44 & $\mathrm{C} 21 \mathrm{H} 2306$ & & & & & & $\begin{array}{l}395.1467[\mathrm{M}+\mathrm{Na}]+\rrbracket \\
767.3038[2 \mathrm{M}+\mathrm{Na}]+\end{array}$ & Unk \\
\hline 105 & 11.79 & $\mathrm{C} 15 \mathrm{H} 1604$ & & & & 261.1127 & 261.1121 & $\begin{array}{l}283.0938[\mathrm{M}+\mathrm{Na}]+\rrbracket \\
243.0996[\mathrm{M}+\mathrm{H}-\mathrm{H} 2 \mathrm{O}]+\end{array}$ & Hes \\
\hline 106 & 12.20 & $\mathrm{C} 16 \mathrm{H} 1006$ & 297.0399 & 297.0394 & $\begin{array}{l}253.0583[\mathrm{M}-\mathrm{H}-\mathrm{CO} 2]-\mathrm{Q} \\
225.0548[\mathrm{M}-\mathrm{H}-\mathrm{CO} 2- \\
\text { CO]- }\end{array}$ & 299.0556 & 299.0589 & & Unk \\
\hline 107 & 12.34 & $\mathrm{C} 2 \mathrm{OH} 2007$ & & & & 373.1287 & 373.1281 & $\begin{array}{l}395.1101[\mathrm{M}+\mathrm{Na}]+\rrbracket \\
767.2318[2 \mathrm{M}+\mathrm{Na}]+\rrbracket \\
343.0810[\mathrm{M}+\mathrm{H}-2 \mathrm{CH} 3]+\rrbracket \\
271.0599[\mathrm{M}+\mathrm{H}-4 \mathrm{CH} 3 \mathrm{O}]+\end{array}$ & Tan \\
\hline 108 & 12.38 & С38H54019 & 813.3181 & 813.3162 & $\begin{array}{l}\text { 859.3215[M+HCOO]- } \rrbracket \\
651.2628[\mathrm{M}-\mathrm{H}-\mathrm{Glc}]-\rrbracket \\
489.2126[\mathrm{M}-\mathrm{H}-2 \mathrm{Glc}]-\rrbracket \\
327.1615[\mathrm{M}-\mathrm{H}-3 \mathrm{Glc}]-\rrbracket \\
\text { 283.1715[M-H-3Glc- } \\
\text { CO2]- }\end{array}$ & 815.3338 & 815.3289 & 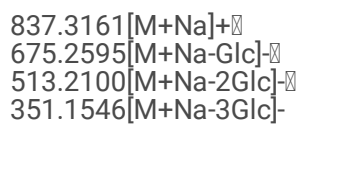 & Cror \\
\hline 109 & 12.52 & $\mathrm{C} 55 \mathrm{H} 74028$ & 1181.4228 & 1181.4275 & $\begin{array}{l}\text { 857.3233[M-H-2Glc]- } ₫ \\
651.2679[\mathrm{M}-\mathrm{H}-3 \mathrm{Glc}- \\
\text { CO2]- }\end{array}$ & & & & Unk \\
\hline 110 & 12.52 & $\mathrm{C} 21 \mathrm{H} 2406$ & 371.1495 & 371.1490 & & & & & Unk \\
\hline 111 & 12.52 & $\mathrm{C} 19 \mathrm{H} 1806$ & & & & 343.1182 & 343.1177 & $\begin{array}{l}365.0988[\mathrm{M}+\mathrm{Na}]+\rrbracket \\
707.2096[2 \mathrm{M}+\mathrm{Na}]+\rrbracket \\
313.0688[\mathrm{M}+\mathrm{H}-2 \mathrm{CH} 3]+\rrbracket \\
285.0688[\mathrm{M}+\mathrm{H}-2 \mathrm{CH} 3-\mathrm{CO}]+\rrbracket \\
153.0166[\mathrm{M}+\mathrm{H}-\mathrm{C} 9 \mathrm{H} 8 \mathrm{O}-\mathrm{CO}- \\
2 \mathrm{CH}]+\end{array}$ & $\begin{array}{l}31, \text {, } \\
\text { Tetr }\end{array}$ \\
\hline
\end{tabular}




\begin{tabular}{|c|c|c|c|c|c|c|c|c|c|}
\hline 112 & 12.55 & $\mathrm{C} 26 \mathrm{H} 3008$ & 469.1862 & 469.1862 & $515.1919[\mathrm{M}+\mathrm{HCOO}]-$ & 471.2019 & 471.2014 & $\begin{array}{l}493.1789[\mathrm{M}+\mathrm{Na}]+\square \\
963.3779[2 \mathrm{M}+\mathrm{Na}]+\end{array}$ & Lim \\
\hline 113 & 12.59 & $\mathrm{C} 17 \mathrm{H} 1406$ & 313.0712 & 313.0712 & 269.0457[M-H-CO2]- & 315.0869 & 315.0863 & & Unk \\
\hline 114 & 12.71 & $\mathrm{C} 48 \mathrm{H} 60022$ & 987.3498 & 987.3476 & 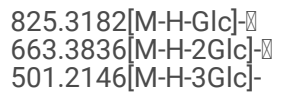 & & & 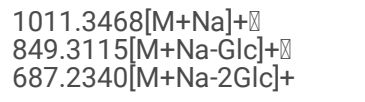 & Unk \\
\hline 115 & 12.75 & $\mathrm{C} 28 \mathrm{H} 36010$ & 531.2230 & 531.2228 & & 533.2387 & 533.2380 & $555.2204[\mathrm{M}+\mathrm{Na}]+$ & Non \\
\hline 116 & 12.78 & $\mathrm{C} 48 \mathrm{H} 78019$ & 957.5059 & 957.5042 & 1003.509[M+HCOO]- & 959.5216 & 959.5223 & $981.4979[\mathrm{M}+\mathrm{Na}]+$ & Unk \\
\hline 117 & 12.79 & $\mathrm{C} 15 \mathrm{H} 806$ & 283.0243 & 283.0239 & $\begin{array}{l}\text { 239.0342[M-H-CO2]-区 } \\
183.0439[\mathrm{M}-\mathrm{H}-\mathrm{CO} 2- \\
\text { 2CO]- }\end{array}$ & 285.0399 & 285.0393 & & Rhe \\
\hline 118 & 12.79 & $\mathrm{C} 18 \mathrm{H} 1607$ & 343.0818 & 343.0816 & & 343.0974 & 343.0967 & & Unk \\
\hline 119 & 12.80 & $\mathrm{C} 32 \mathrm{H} 44014$ & 651.2653 & 651.2646 & $\begin{array}{l}327.1689[\mathrm{M}-\mathrm{H}-2 \mathrm{Glc}]-\mathbb{} \\
283.1749[\mathrm{M}-\mathrm{H}-2 \mathrm{Glc}- \\
\text { CO2]- }\end{array}$ & & & $675.2632[\mathrm{M}+\mathrm{Na}]+$ & Cror \\
\hline 120 & 12.83 & $\mathrm{C} 48 \mathrm{H} 78018$ & 941.5110 & 941.5092 & $987.514[\mathrm{M}+\mathrm{HCOO}]-$ & 943.5266 & 943.5270 & $965.5088[\mathrm{M}+\mathrm{Na}]+$ & Unk \\
\hline 121 & 12.83 & $\mathrm{C} 21 \mathrm{H} 22 \mathrm{O} 8$ & & & & 403.1399 & 403.1393 & $\begin{array}{l}425.1209[\mathrm{M}+\mathrm{Na}]+\rrbracket \\
827.2540[2 \mathrm{M}+\mathrm{Na}]+\square \\
388.1130[\mathrm{M}+\mathrm{H}-\mathrm{CH}]+\square \\
373.0912[\mathrm{M}+\mathrm{H}-2 \mathrm{CH}]+\square \\
358.0676[\mathrm{M}+\mathrm{H}-3 \mathrm{CH} 3]+\end{array}$ & Not \\
\hline 122 & 12.85 & $\mathrm{C} 32 \mathrm{H} 44014$ & 651.2653 & 651.2658 & $\begin{array}{l}\text { 327.1598[M-H-2Glc]- } ₫ \\
283.0238[\mathrm{M}-\mathrm{H}-2 \mathrm{Glc}- \\
\text { CO2]- }\end{array}$ & & & $675.2595[\mathrm{M}+\mathrm{Na}]+$ & Cror \\
\hline 123 & 12.91 & C47H76017 & 911.5004 & 911.4994 & $957.5037[\mathrm{M}+\mathrm{HCOO}]-$ & & & $935.4932[\mathrm{M}+\mathrm{Na}]+$ & Unk \\
\hline 124 & 13.05 & $\mathrm{C} 22 \mathrm{H} 2409$ & & & & 433.1499 & 433.1501 & 418.1250[M+H-CH3]+ & $\begin{array}{l}31,4 \\
\text { Hep }\end{array}$ \\
\hline 125 & 13.07 & $\mathrm{C} 57 \mathrm{H} 62 \mathrm{O} 3$ & 793.4621 & 793.4614 & 839.4663[M+HCOO]- & 795.4777 & 795.4777 & 817.4599[M+Na]+ & Unk \\
\hline 126 & 13.08 & $\mathrm{C} 17 \mathrm{H} 2604$ & 293.1753 & 293.1755 & $\begin{array}{l}264.9948[\mathrm{M}-\mathrm{H}-\mathrm{CO}]-\rrbracket \\
221.1583[\mathrm{M}-\mathrm{H}-\mathrm{CO}- \\
\text { CO2]- }\end{array}$ & & & & Unk \\
\hline 127 & 13.13 & $\mathrm{C} 21 \mathrm{H} 22 \mathrm{O}$ & & & & 419.1342 & 419.1342 & $\begin{array}{l}441.1161[\mathrm{M}+\mathrm{Na}]+\rrbracket \\
859.2434[2 \mathrm{M}+\mathrm{Na}]+\rrbracket \\
404.1098[\mathrm{M}+\mathrm{H}-\mathrm{CH} 3]+\rrbracket \\
389.0582[\mathrm{M}+\mathrm{H}-2 \mathrm{CH}]+\end{array}$ & $\begin{array}{l}3^{\prime}, 4 \\
\text { Hex }\end{array}$ \\
\hline 128 & 13.17 & $\mathrm{C} 13 \mathrm{H} 24012$ & & & & 373.1346 & 373.1352 & $\begin{array}{l}395.1116[\mathrm{M}+\mathrm{Na}]+\rrbracket \\
767.2371[2 \mathrm{M}+\mathrm{Na}]+\end{array}$ & Unk \\
\hline 129 & 13.22 & $\mathrm{C} 17 \mathrm{H} 2605$ & 309.1702 & 309.1706 & 146.9664[M-H-Glc]- & & & & Unk \\
\hline 130 & 13.22 & С30H50012 & 601.3224 & 601.3227 & 657.0352[M+HCOO]- & & & 625.3192[M+Na]+ & Unk \\
\hline 131 & 13.24 & $\mathrm{C} 13 \mathrm{H} 2004$ & 239.1283 & 239.1286 & 195.1385[M-H-CO2]- & & & & Unk \\
\hline 132 & 13.33 & $\mathrm{C} 2 \mathrm{OH} 2008$ & & & & 389.1236 & 389.1232 & $\begin{array}{l}411.1052[\mathrm{M}+\mathrm{Na}]+\rrbracket \\
799.2224[2 \mathrm{M}+\mathrm{Na}]+\rrbracket \\
374.1299[\mathrm{M}+\mathrm{H}-\mathrm{CH} 3]+\rrbracket \\
359.0751[\mathrm{M}+\mathrm{H}-2 \mathrm{CH}]+\end{array}$ & $\begin{array}{l}5-\mathrm{H} ! \\
5^{\prime}- \\
\text { pen }\end{array}$ \\
\hline 133 & 13.33 & $\mathrm{C} 30 \mathrm{H} 4805$ & 487.3423 & 487.3433 & 469.0117[M-H-H2O]- & & & & Eruk \\
\hline 134 & 13.38 & $\mathrm{C} 24 \mathrm{H} 3006$ & & & & 415.2121 & 415.2118 & 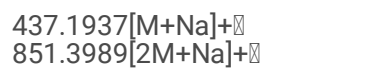 & Unk \\
\hline 135 & 13.46 & $\mathrm{C} 15 \mathrm{H} 1005$ & 269.0450 & 269.0453 & $\begin{array}{l}241.0499[\mathrm{M}-\mathrm{H}-\mathrm{CO}]-\mathbb{\Omega} \\
225.0549[\mathrm{M}-\mathrm{H}-\mathrm{CO}-\mathrm{O}]-\end{array}$ & 271.0606 & 271.0601 & & Emc \\
\hline 136 & 13.84 & $\mathrm{C} 13 \mathrm{H} 2808$ & 311.1706 & 311.1686 & & & & & Unk \\
\hline 137 & 13.90 & $\mathrm{C} 24 \mathrm{H} 4008$ & & & & 457.2776 & 457.2801 & & Unk \\
\hline 138 & 14.08 & $\mathrm{C} 27 \mathrm{H} 54013$ & 585.3486 & 585.3472 & $\begin{array}{l}\text { 233.1061[M-H-GlC- } \\
\text { Rha-CO2]- }\end{array}$ & & & & Unk \\
\hline 139 & 14.11 & C57H9806 & & & & 879.7442 & 879.7438 & 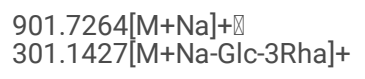 & Unk \\
\hline 140 & 14.16 & $\mathrm{C} 21 \mathrm{H} 2802$ & 311.2011 & 311.2020 & 149.0965[M-H-Glc]- & & & & Unk \\
\hline 141 & 14.22 & $\mathrm{C} 15 \mathrm{H} 22 \mathrm{O} 3$ & 249.1491 & 249.1494 & 205.1611[M-H-CO2]- & & & & Unk \\
\hline 142 & 14.29 & $\mathrm{C} 2 \mathrm{OH} 3408$ & & & & 403.2332 & 403.2324 & $425.2149[\mathrm{M}+\mathrm{Na}]+\nabla$ & Unk \\
\hline
\end{tabular}

Page $11 / 28$ 


\begin{tabular}{|c|c|c|c|c|c|c|c|c|c|}
\hline 143 & 14.32 & $\mathrm{C} 14 \mathrm{H} 3008$ & 325.1862 & 325.1846 & & & & & Unk \\
\hline 144 & 14.86 & $\mathrm{C} 24 \mathrm{H} 34 \mathrm{O} 2$ & 353.2481 & 353.2481 & $\begin{array}{l}325.1841[\mathrm{M}-\mathrm{H}-\mathrm{CO}]-\mathbb{} \\
281.2469[\mathrm{M}-\mathrm{H}-\mathrm{CO}- \\
\mathrm{CO} 2]-\end{array}$ & & & & Unk \\
\hline 145 & 14.98 & C57H9806 & & & & 879.7442 & 879.7438 & $901.7277[\mathrm{M}+\mathrm{Na}]+$ & Unk \\
\hline 146 & 15.01 & C15H32O8 & 339.2019 & 339.1994 & & & & & Unk \\
\hline 147 & 15.49 & $\mathrm{C} 21 \mathrm{H} 2405$ & 355.1545 & 355.1574 & 311.2009[M-H-CO2]- & & & & Unk \\
\hline
\end{tabular}

* represented the standard compound.

\subsubsection{Identification of compounds in Gardeniae Fructus}

A total of 61 compounds were obtained from ZZ, of which were 30 known compounds. ZZ contained a large number of iridoid glucosides, including gardoside, shanzhiside, genipin-1- $\beta$-D-gentiobioside, geniposide, and gardenoside. Crocins, including crocin-I and crocin-II, were deemed the main active compounds in ZZ[30]. Geniposide, the most abundant iridoid glucosides in ZZDHD, and crocin-I, the most abundant crocin, were used to characterize the fragmentation pathways, which are shown in Fig. A1.

The [M-H]- ion and the typical ions in the $\mathrm{MS}^{2}$ spectra of peak $46\left(\mathrm{t}_{\mathrm{R}}=4.68\right)$, namely, m/z 387.1296 [M-H]-, 433.1408 [M + HCOO]-, and 225.0763 [M-H-Glc]- were all $162 \mathrm{Da}$ less compared to those of peak $41\left(t_{R}=4.33\right)$, demonstrating that they are cleaved at the same site. By comparing the retention time values and mass data with those of the reference[26], peak 46 was therefore characterized as geniposide, and peak 41 was regarded as genipin-1-0-3-D-gentiobioside.

Peak $65\left(t_{R}=5.58\right)$ showed the $[M-H]$ - ion at $\mathrm{m} / \mathrm{z}$ 975.3709, which was the correlative elemental composition of $\mathrm{C}_{44} \mathrm{H}_{63} \mathrm{O}_{24}{ }^{-}$. After losing two molecules of glucose and four molecules of glucose, the $\mathrm{MS}^{2}$ fragment ions were generated as 651.2679 [M-H-2Glc]- and 327.1615 [M-H-2Glc-2Glc]-, respectively. Resulting from loss of one molecule of $\mathrm{CO}_{2}$, the [M-H-2Glc-2Glc- $\left.\mathrm{CO}_{2}\right]$ - ion at $\mathrm{m} / \mathrm{z} 283.1700$ was observed. Based on the molecular weight and multi-stage information provided by $\mathrm{MS}^{\mathrm{n}}$ and previously published literature[27], peak 65 was inferred as crocin-I. Based on these fragmentation patterns, other crocins were accurately identified.

\subsubsection{Identification of compounds in Fructus Aurantii Immaturus}

In total, 51 compounds derived from ZS were detected in ZZDHD, including 26 known compounds. ZS contained large number of flavonoids, including dihydroflavones and isoflavones, such as narirutin, naringin, hesperidin, and neohesperidin. Their fragmentation patterns were are in Fig. A2.

The [M-H]- ion of peak $63\left(\mathrm{t}_{\mathrm{R}}=5.52\right)$ was $\mathrm{m} / \mathrm{z} 579.1740\left(\mathrm{C}_{27} \mathrm{H}_{31} \mathrm{O}_{14}{ }^{-}\right)$. The $\mathrm{MS}^{2}$ spectra exhibited characteristic product ions, such as $\left[\mathrm{M}-\mathrm{H}-\mathrm{C}_{8} \mathrm{H}_{8} \mathrm{O}\right]-(\mathrm{m} / \mathrm{z}$ 459.1146), [M-H-Rha-Glc]- (m/z 271.0616), and [M-H-Rha-Glc- $\left.\mathrm{C}_{8} \mathrm{H}_{8} \mathrm{O}\right]-(\mathrm{m} / \mathrm{z} 151.0028)$, thus the compounds were deduced as narirutin. Naringin had a similar cleavage pattern to narirutin, and according to the literature[23], peak $66\left(t_{R}=5.62\right)$ was identified as naringin.

Peak $68\left(\mathrm{t}_{\mathrm{R}}=5.73\right)$ displayed [M-H]- ion at $\mathrm{m} / \mathrm{z} 609.1841\left(\mathrm{C}_{28} \mathrm{H}_{34} \mathrm{O}_{15}{ }^{-}\right)$and its $\mathrm{MS}^{2}$ spectra exhibited at m/z 301.0722 [M-H-Rha-Glc]- and 151.0031 [M-H-Rha$\mathrm{Glc}_{2} \mathrm{C}_{9} \mathrm{H}_{10} \mathrm{O}_{2}$ ]. Based on the fragmentation information and previously published studies[23], peak 68 was primarily identified as hesperidin.

\subsubsection{Identification of compounds in Sojae Semen Praeparatum}

The major active constituents of DDC were isoflavones[24]. It contained 24 compounds, including eight known compounds, such as daidzin, daidzein, and genistein[31].

In the positive mode, peak $48\left(t_{R}=4.86\right)$ displayed the $[M+H]+$ ion at $m / z 417.1214$ and the $[M+N a]+$ ion at $m / z$ 439.1006. The $M S^{2}$ spectrum provided a diagnostic fragment ion at $\mathrm{m} / \mathrm{z} 255.0612$, resulting from the loss of one molecule of glucose. It displayed the [M-H]- ion at $\mathrm{m} / \mathrm{z} 417.1183$ and the [M $+\mathrm{HCOO}$ ion at $\mathrm{m} / \mathrm{z}$ 461.1094. In addition, a main MS/MS pattern at $\mathrm{m} / \mathrm{z} 253.0505$ was observed for peak 48 , corresponding to the loss of a glucose residue.

Consequently, peak 48 was unambiguously identified as daidzin.

Peak $82\left(\mathrm{t}_{\mathrm{R}}=6.41\right)$ exhibited the $[\mathrm{M}-\mathrm{H}]$ - ion at $\mathrm{m} / \mathrm{z} 253.0500$ with a molecular formula of $\mathrm{C}_{15} \mathrm{H}_{9} \mathrm{O}_{4}{ }^{-}$; it showed the quasi-molecular ion [M $\left.+\mathrm{H}\right]+$ ion at $\mathrm{m} / \mathrm{z}$ 255.0659 and the sodium adduct ion $[\mathrm{M}+\mathrm{Na}]+$ ion at $\mathrm{m} / \mathrm{z} 277.0447$, which was definitely assigned as daidzein.

Peak $93\left(t_{R}=7.91\right)$ had the characteristic fragment of ion $[M-H]-$ at $m / z 269.0443$, representing the corresponding elemental composition of $C_{15} \mathrm{H}_{10} \mathrm{O}_{5}$, and its positive ion was $271.0597[\mathrm{M}+\mathrm{H}]+$ and $541.0952[2 \mathrm{M}+\mathrm{H}]+$. It was tentatively identified as genistein.

\subsubsection{Identification of compounds in Rhei Radix et Rhizoma}

DH contained 25 compounds, including 14 known constituents. Anthraquinones, such as rhein and emodin, were likely detected in ZZDHD as they are compounds typically found in $\mathrm{DH}[19]$. Anthraquinones generally have strong MS response in negative ion mode and weak MS response in positive ion mode. 
Peak $117\left(t_{R}=12.79\right)$ and $135\left(t_{R}=13.46\right)$ were recognized as rhein and emodin, respectively, and they were used to characterize the fragmentation pathways (Fig. A3). In the negative ion mode, peak 117 showed [M-H]- at m/z 283.0239, and then generated a fragment ion at $\mathrm{m} / \mathrm{z} 239.0342$, owing to the loss of a $\mathrm{CO}_{2}$ residue. Subsequently, the fragment $\mathrm{m} / \mathrm{z} 211.0391$ was yielded due to the loss of CO from 239.0342. Lastly the ion further lost one molecule of CO to produce an ion at $\mathrm{m} / \mathrm{z}$ 183.0441. Based upon these data and the literature[28], the compound was rhein. Peak 135 showed [M-H]- at $\mathrm{m} / \mathrm{z} 269.0453$, and then generated fragments with $\mathrm{m} / \mathrm{z} 241.0499$ and $\mathrm{m} / \mathrm{z} 225.0549$, which was due to the loss of $\mathrm{CO}$ and $\mathrm{CO}_{2}$. Based upon these data and previously published research[29], peak 135 was determined to be emodin. Other constituents of anthraquinones in DH were identified using this fragment information (Table 2).

\subsection{UPLC-DAD quantification}

\subsubsection{Method validation}

Quantitative method validation for the established UPLC-DAD analysis was performed for linearity, precision, stability, repeatability, and recovery, as shown in Table 3. All correlation coefficient values $(r>0.999)$ demonstrated a good linear relationship between the analyte concentrations and their peak areas within the relatively wide test ranges. The relative standard diviation (RSD) of precision with 15 analytes ranged from $0.29-3.97 \%$. The RSDs for stability were less than $4.22 \%$. As for repeatability, the RSDs were not more than $4.36 \%$. The developed method also had suitable accuracy with spike recovery of $95.35-107.95 \%$ for all analytes. These results demonstrated that the proposed quantitative UPLC-DAD method was linear, precise, stable, repeatable, and sufficiently accurate for simultaneous determination of fifteen compounds in ZZDHD samples.

Table 3

Method validation for quantitative determination of fifteen compounds.

\begin{tabular}{|c|c|c|c|c|c|c|c|c|c|}
\hline \multirow[t]{2}{*}{$\begin{array}{l}\text { Reference } \\
\text { Standards }\end{array}$} & \multicolumn{3}{|c|}{ Working Standard Cruve } & \multirow{2}{*}{$\begin{array}{l}\text { Precision } \\
\text { (RSD, \%, n } \\
=6 \text { ) }\end{array}$} & \multirow{2}{*}{$\begin{array}{l}\text { Stability } \\
(24 \mathrm{~h}, \\
\text { RSD, \%) }\end{array}$} & \multirow{2}{*}{$\begin{array}{l}\text { Repeatability } \\
\text { (RSD, \%, n = } \\
6 \text { ) }\end{array}$} & \multicolumn{3}{|c|}{$\begin{array}{l}\text { Spike Recovery } \\
\text { (Mean (RSD), \%, n=3) }\end{array}$} \\
\hline & Equation & $\mathbf{r}$ & $\begin{array}{l}\text { Linear Range } \\
(\mathrm{mg} / \mathrm{mL})\end{array}$ & & & & Low & Middle & High \\
\hline G1 & $\begin{array}{l}y=1027.7 x+ \\
11477\end{array}$ & 0.9998 & $\begin{array}{l}21.56 \sim \\
1380.00\end{array}$ & 1.43 & 1.61 & 1.45 & $100.56(1.31)$ & $102.68(1.08)$ & $94.35(1.78)$ \\
\hline G2 & $\begin{array}{l}y=2446 x+ \\
157377\end{array}$ & 0.9996 & $\begin{array}{l}69.22 \sim \\
4430.00\end{array}$ & 0.81 & 0.34 & 1.67 & $101.26(0.62)$ & $98.20(0.64)$ & $104.39(0.42)$ \\
\hline daidzin & $\begin{array}{l}y=12059 x+ \\
13005\end{array}$ & 0.9998 & $1.01 \sim 64.50$ & 1.46 & 1.07 & 1.36 & $100.34(0.57)$ & 101.48(1.77) & $100.87(0.17)$ \\
\hline narirutin & $\begin{array}{l}y=5640.8 x+ \\
32911\end{array}$ & 0.9998 & $8.03 \sim 514.00$ & 1.40 & 0.97 & 0.65 & $99.92(2.47)$ & $99.01(2.10)$ & 107.07(1.88) \\
\hline naringin & $\begin{array}{l}y=7126.7 x- \\
255950\end{array}$ & 0.9991 & $\begin{array}{l}70.63 \sim \\
2260.00\end{array}$ & 1.11 & 1.74 & 1.72 & $100.44(4.71)$ & $99.49(0.64)$ & $106.38(0.36)$ \\
\hline crocin-I & $\begin{array}{l}y=22492 x+ \\
41294\end{array}$ & 1.0000 & $5.94 \sim 380.00$ & 0.40 & 0.80 & 1.91 & $102.76(1.22)$ & $100.40(5.31)$ & $105.58(1.28)$ \\
\hline hesperidin & $\begin{array}{l}y=5206.5 x+ \\
18349\end{array}$ & 0.9996 & $\begin{array}{l}12.72 \sim \\
814.00\end{array}$ & 0.73 & 1.44 & 1.60 & $95.24(0.40)$ & $105.36(2.56)$ & $99.15(2.07)$ \\
\hline neohesperidin & $\begin{array}{l}y=8828.7 x- \\
243244\end{array}$ & 0.9992 & $\begin{array}{l}57.81 \sim \\
1387.50\end{array}$ & 1.14 & 0.60 & 1.91 & 104.65(7.11) & $101.19(0.38)$ & $104.66(2.30)$ \\
\hline daidzein & $\begin{array}{l}y=18858 x+ \\
7509.3\end{array}$ & 0.9998 & $0.64 \sim 41.08$ & 1.95 & 1.54 & 0.99 & $99.17(2.49)$ & $98.66(1.94)$ & $105.20(1.83)$ \\
\hline genistein & $\begin{array}{l}y=23742 x+ \\
8741.3\end{array}$ & 0.9998 & $0.61 \sim 39.28$ & 1.64 & 1.79 & 1.10 & $102.75(0.32)$ & $102.06(0.73)$ & $106.41(2.35)$ \\
\hline rhein & $\begin{array}{l}y=5393 x+ \\
4241.3\end{array}$ & 0.9996 & $1.11 \sim 71.00$ & 0.92 & 0.89 & 1.37 & $95.19(1.97)$ & 107.94(1.79) & $107.94(0.35)$ \\
\hline crocetin & $\begin{array}{l}y=27114 x+ \\
401.9\end{array}$ & 0.9998 & $0.02 \sim 1.50$ & 3.97 & 4.22 & 4.36 & $101.38(0.97)$ & 103.16(1.07) & $100.01(3.60)$ \\
\hline emodin & $\begin{array}{l}y=5871.2 x+ \\
626.5\end{array}$ & 0.9999 & $0.19 \sim 12.45$ & 1.71 & 0.95 & 1.46 & $99.47(1.48)$ & $100.17(0.73)$ & $107.09(0.99)$ \\
\hline chrysophanol & $\begin{array}{l}y=8476 x+ \\
514.26\end{array}$ & 0.9999 & $0.10 \sim 6.64$ & 0.29 & 0.52 & 1.05 & $106.69(0.52)$ & $107.72(2.45)$ & $96.96(2.50)$ \\
\hline physcion & $\begin{array}{l}y=8423.2 x+ \\
108.53\end{array}$ & 0.9998 & $0.03 \sim 1.66$ & 0.68 & 0.73 & 1.03 & $101.16(1.68)$ & $98.19(1.30)$ & $102.91(2.80)$ \\
\hline
\end{tabular}

\subsubsection{Analysis of content determination}

This study investigated the differences in the chemical compounds of ZZDHD prepared following two different boiling methods. Representative chromatograms of one sample (S1) and reference standards at $254 \mathrm{~nm}, 283 \mathrm{~nm}$, and $440 \mathrm{~nm}$ are presented in Fig. 3. A total of 12 batches of ZZDHD samples 
from the preparation methods were collected, prepared, and analyzed as described above. Fifteen chemical substances. Specifically, G1, G2, daidzin, narirutin, naringin, crocin-I, hesperidin, neohesperidin, daidzein, genistein, rhein, crocetin, emodin, chrysophanol, and physcion (Fig. 1) were chosen as the reference standards for quantitation. The content of each marker compound was determined at its maximum absorption wavelength, using the external standard method. The quantitative data for the marker compounds are presented in Table 4. A slightly variation in the content of other marker compounds (RSD < 30\%) was observed, except for the physcion content. A relatively high content of $\mathrm{G} 2$, naringin, and neohesperidin were present in both the single- co-boiled samples. As shown in Fig. 4, the difference was statistically significant between the contents of $G 2$, emodin, and chrysophanol in $C B$ and $S B(P<0.05)$, and the difference was highly statistically significantly different between daidzin, physcion $(p<0.01)$, and crocin-I $(p<0.001)$. There were no significant differences among the other nine compounds.

Table 4

Quantitative determination results of 15 reference standards in Zhi-Zi-Da-Huang decoction $(\mu \mathrm{g} / \mathrm{g}, \mathrm{n}$

\begin{tabular}{|llllll|}
\hline Reference Standards & $\mathrm{t}_{\mathrm{R}} / \mathrm{min}$ & $\mathrm{CB}$ & $\mathrm{SB}$ & Source & $\mathrm{RSD} / \%$ \\
\hline $\mathrm{G} 1$ & 7.53 & $3940.75 \pm 640.79$ & $3804.15 \pm 260.04$ & $\mathrm{ZZ}$ & 5.89 \\
\hline $\mathrm{G} 2$ & 8.03 & $10946.08 \pm 516.87$ & $10186.12 \pm 283.51$ & $\mathrm{ZZ}$ & 5.32 \\
\hline daidzin & 8.45 & $169.2 \pm 5.69$ & $183.4 \pm 8.76$ & $\mathrm{DDC}$ & 9.05 \\
\hline narirutin & 9.29 & $1001.79 \pm 151.21$ & $923.96 \pm 75.49$ & $\mathrm{ZS}$ & 12.18 \\
\hline naringin & 9.44 & $11492.88 \pm 349.95$ & $11580.58 \pm 942.6$ & $\mathrm{ZS}$ & 10.24 \\
\hline crocin-I & 9.45 & $950.99 \pm 48.56$ & $994.84 \pm 79.12$ & $\mathrm{ZZ}$ & 12.56 \\
\hline hesperidin & 9.59 & $2078.45 \pm 73.72$ & $1903.24 \pm 260.08$ & $\mathrm{ZS}$ & 6.85 \\
\hline neohesperidin & 9.76 & $9585.49 \pm 369.15$ & $9340.64 \pm 1201$ & $\mathrm{ZS}$ & 16.31 \\
\hline daidzein & 10.54 & $104.82 \pm 2.49$ & $106.7 \pm 16.27$ & $\mathrm{DDC}$ & 5.80 \\
\hline genistein & 12.85 & $97.66 \pm 4.31$ & $102.54 \pm 13.37$ & $\mathrm{DDC}$ & 10.53 \\
\hline rhein & 16.29 & $206.1 \pm 49.21$ & $203.28 \pm 4.99$ & $\mathrm{DH}$ & 9.80 \\
\hline crocetin & 16.69 & $3.93 \pm 0.28$ & $1.16 \pm 0.28$ & $\mathrm{ZZ}$ & 18.22 \\
\hline emodin & 17.02 & $36.94 \pm 8.36$ & $45.92 \pm 2.68$ & $\mathrm{DH}$ & 13.84 \\
\hline chrysophanol & 17.72 & $18.69 \pm 2.81$ & $22.12 \pm 1.6$ & $\mathrm{DH}$ & 12.40 \\
\hline physcion & 18.02 & $4.65 \pm 0.41$ & $5.64 \pm 0.37$ & $\mathrm{DH}$ & 57.73 \\
\hline
\end{tabular}

\subsection{UPLC-DAD fingerprint}

\subsubsection{Method validation}

A comprehensive validation of the established UPLC fingerprint method was carried out, including assessments of precision, repeatability, and stability, and the results were summarized in Table 5 . The intra- and inter-day variations of all 23 common peaks (RSDs) were within $0.02-0.19 \%$ and $0.05-0.32 \%$ for the relative retention time (RRT), as well as $1.62-4.73 \%$ and $1.83-4.87 \%$ for the relative peak area (RPA), respectively.

In the precision, repeatability, and stability tests, RSDs of the RPA from all 30 common peaks were less than $3.80 \%$, 2.74\%, and $2.54 \%$, respectively (Table 5). These results showed that the UPLC fingerprinting method was applicable for the qualitative analysis of ZZDHD. 
Table 5

Method validation of common peaks in Zhi-Zi-Da-Huang decoction.

\begin{tabular}{|c|c|c|c|c|c|c|c|c|c|}
\hline \multirow[t]{2}{*}{ No. } & \multirow[t]{2}{*}{$t_{R} / \min$} & \multicolumn{3}{|l|}{ RSD/\% } & \multirow[t]{2}{*}{ No. } & \multirow[t]{2}{*}{$t_{R} / \min$} & \multicolumn{3}{|l|}{ RSD/\% } \\
\hline & & Precision & Repeatability & Stability & & & Precision & Repeatability & Stability \\
\hline$A 1^{d}$ & 1.058 & 2.21 & 2.02 & 2.04 & $B 4^{e}$ & 9.569 & 0.73 & 1.60 & 1.30 \\
\hline$A 2^{d}$ & 1.979 & 1.41 & 2.17 & 1.35 & $B 5^{\mathrm{e}}$ & 9.720 & 1.14 & 1.91 & 0.60 \\
\hline$A 3^{d}$ & 2.659 & 1.98 & 1.42 & 0.85 & $B 6^{e}$ & 11.58 & 0.42 & 1.51 & 0.66 \\
\hline $\mathrm{A} 4^{\mathrm{d}}$ & 3.292 & 1.19 & 1.61 & 2.54 & $C 1^{f}$ & 3.639 & 0.46 & 2.20 & 0.67 \\
\hline$A 5^{d}$ & 5.682 & 3.80 & 1.52 & 1.39 & $C 2^{f}$ & 9.439 & 0.40 & 1.91 & 0.71 \\
\hline$A 6^{d}$ & 7.525 & 1.43 & 1.53 & 1.11 & $C 3^{f}$ & 9.542 & 1.63 & 2.31 & 2.15 \\
\hline$A 7^{d}$ & 7.747 & 2.49 & 1.67 & 1.52 & $C 4^{f}$ & 9.984 & 2.23 & 1.48 & 1.28 \\
\hline$A 8^{d}$ & 8.030 & 0.81 & 1.67 & 0.54 & $C 5^{f}$ & 14.853 & 2.75 & 2.55 & 1.33 \\
\hline$A 9^{d}$ & 8.431 & 1.46 & 1.36 & 0.69 & $C 6^{f}$ & 15.175 & 0.34 & 1.57 & 0.67 \\
\hline$A 10^{d}$ & 10.253 & 1.57 & 1.65 & 1.48 & $C 7^{f}$ & 15.396 & 0.42 & 2.06 & 0.59 \\
\hline$A 11^{d}$ & 10.487 & 1.95 & 0.99 & 1.30 & $C 8^{f}$ & 16.283 & 0.92 & 1.60 & 0.91 \\
\hline$A 12^{d}$ & 12.591 & 1.64 & 1.10 & 1.35 & $C 9^{f}$ & 16.693 & 0.55 & 1.75 & 1.68 \\
\hline $\mathrm{B} 1^{\mathrm{e}}$ & 1.339 & 2.91 & 2.74 & 2.42 & $\mathrm{C} 10^{f}$ & 17.018 & 1.71 & 1.55 & 1.09 \\
\hline$B 2^{e}$ & 9.281 & 1.40 & 0.65 & 0.72 & $\mathrm{C} 11^{f}$ & 17.716 & 0.29 & 1.05 & 0.45 \\
\hline$B 3^{e}$ & 9.426 & 1.11 & 1.72 & 1.71 & $\mathrm{C} 12^{f}$ & 18.020 & 0.69 & 1.03 & 0.91 \\
\hline
\end{tabular}

d represented the peak recorded at $254 \mathrm{~nm}$; e represented the peak recorded at $280 \mathrm{~nm} ; \mathrm{f}$ represented the peak recorded at $440 \mathrm{~nm}$; $\mathrm{t}_{\mathrm{R}}$ was retention times; $R P A$ was relative peak areas.

\subsubsection{Fingerprinting analysis}

Twelve batches of samples were analyzed under optimized conditions. As shown in Fig. 5, all samples showed similar chromatographic profiles. Peaks existing in all samples were designated as common peaks, and 30 common peaks were found, including 12 common peaks identified at 254 nm, six common peaks identified at $280 \mathrm{~nm}$, and 12 common peaks identified at $440 \mathrm{~nm}$. According to the representative standard peak area of the fingerprint calculated by the Similarity Evaluation System Chromatographic Fingerprint of Traditional Chinese Medicine Chromatographic Fingerprint software, the RPA of the ZZDHD samples was calculated. The peak of the representative standard fingerprint was designated as the reference peak (RPA = peak area of characteristic peak/peak area of reference peak). As shown in Table A1, changes in the contents of single- and co-boiled ZZDHD decoctions were analyzed by RPA. The results indicated that the RPA varied dramatically between samples at $254 \mathrm{~nm}$ and $440 \mathrm{~nm}$, and there were two and seven common peaks with an RPA > $15 \%$, respectively, which demonstrated that the content of the single- and the co-boiled was different.

Table A1 Relative peak areas of samples of Zhi-Zi-Da-Huang decoction. 


\begin{tabular}{|c|c|c|c|c|c|c|c|c|c|c|c|c|c|c|c|}
\hline \multirow[t]{2}{*}{ No. } & \multirow[t]{2}{*}{$t_{R} / \min$} & \multirow{2}{*}{$\begin{array}{l}\text { Representative } \\
\text { Peak Area }\end{array}$} & \multicolumn{12}{|l|}{ RPA } & \multirow[t]{2}{*}{ RSD \% } \\
\hline & & & $\mathrm{S} 1$ & $\mathrm{~S} 2$ & S3 & S4 & S5 & S6 & S7 & S8 & s9 & S10 & S11 & $\mathrm{S} 12$ & \\
\hline$A 1^{d}$ & 1.058 & 429846 & 1.069 & 1.010 & 0.999 & 0.916 & 1.005 & 0.982 & 0.915 & 0.925 & 1.061 & 1.057 & 1.016 & 1.045 & 5.58 \\
\hline$A 2^{d}$ & 1.982 & 168667 & 1.026 & 1.024 & 0.929 & 0.944 & 0.953 & 0.926 & 1.067 & 1.026 & 1.079 & 1.080 & 0.970 & 0.977 & 5.73 \\
\hline$A 3^{d}$ & 2.662 & 138705 & 0.886 & 0.917 & 0.839 & 0.845 & 0.818 & 0.804 & 1.219 & 1.179 & 1.145 & 1.159 & 1.092 & 1.096 & 16.11 \\
\hline $\mathrm{A} 4^{\mathrm{d}}$ & 3.296 & 352654 & 1.063 & 1.123 & 0.926 & 0.991 & 0.935 & 0.970 & 1.082 & 1.072 & 0.996 & 0.982 & 0.927 & 0.931 & 6.88 \\
\hline$A 5^{d}$ & 5.689 & 152146 & 1.047 & 0.952 & 0.935 & 0.974 & 0.983 & 0.970 & 1.024 & 1.037 & 0.981 & 0.992 & 1.049 & 1.057 & 4.11 \\
\hline$A 6^{d}$ & 7.527 & 385975 & 1.009 & 1.049 & 1.029 & 0.991 & 1.163 & 1.130 & 0.930 & 0.882 & 0.880 & 0.881 & 1.009 & 1.045 & 9.35 \\
\hline$A 7^{d}$ & 7.748 & 108146 & 1.575 & 1.672 & 0.550 & 0.653 & 0.660 & 0.694 & 1.715 & 1.600 & 0.571 & 0.543 & 0.880 & 0.889 & 48.67 \\
\hline$A 8^{d}$ & 8.030 & 2457611 & 1.048 & 1.045 & 0.974 & 0.972 & 1.092 & 1.078 & 0.968 & 0.961 & 0.984 & 0.987 & 0.952 & 0.940 & 5.14 \\
\hline$A 9^{d}$ & 8.431 & 220362 & 0.922 & 0.974 & 0.948 & 0.961 & 0.959 & 0.931 & 1.012 & 0.990 & 1.086 & 1.119 & 1.038 & 1.060 & 6.35 \\
\hline $\mathrm{A} 10^{\mathrm{d}}$ & 10.248 & 168950 & 1.039 & 0.869 & 1.011 & 0.993 & 0.979 & 0.971 & 1.001 & 0.982 & 1.023 & 1.063 & 1.027 & 1.039 & 4.97 \\
\hline $\mathrm{A} 11^{\mathrm{d}}$ & 10.485 & 211302 & 0.963 & 1.025 & 0.931 & 0.953 & 1.001 & 1.012 & 0.909 & 0.900 & 1.202 & 1.226 & 0.951 & 0.926 & 10.74 \\
\hline $\mathrm{A} 12^{\mathrm{d}}$ & 12.592 & 217763 & 0.983 & 0.962 & 0.868 & 0.927 & 1.029 & 1.011 & 0.936 & 0.903 & 1.191 & 1.195 & 0.987 & 1.007 & 10.17 \\
\hline$B 1^{e}$ & 1.340 & 446235 & 1.013 & 1.006 & 0.833 & 0.823 & 1.191 & 1.178 & 1.014 & 0.898 & 0.906 & 1.044 & 1.066 & 1.028 & 11.84 \\
\hline$B 2^{\mathrm{e}}$ & 9.279 & 408437 & 1.198 & 1.069 & 0.911 & 0.920 & 0.960 & 0.922 & 1.037 & 0.895 & 0.923 & 1.063 & 1.059 & 1.044 & 9.25 \\
\hline $\mathrm{B}^{\mathrm{e}}$ & 9.423 & 7728449 & 0.969 & 0.955 & 1.015 & 0.995 & 1.016 & 0.979 & 1.080 & 0.897 & 0.906 & 1.052 & 1.057 & 1.079 & 6.17 \\
\hline$B 4^{e}$ & 9.565 & 1135465 & 1.018 & 0.984 & 1.040 & 1.078 & 1.084 & 1.071 & 1.009 & 0.782 & 0.769 & 1.060 & 1.060 & 1.044 & 10.89 \\
\hline$B 5^{\mathrm{e}}$ & 9.716 & 7405738 & 0.982 & 0.972 & 1.050 & 1.045 & 1.014 & 1.004 & 1.092 & 0.782 & 0.809 & 1.072 & 1.079 & 1.099 & 10.43 \\
\hline$B 6^{e}$ & 11.580 & 372710 & 0.942 & 0.921 & 1.018 & 1.024 & 0.982 & 0.965 & 1.076 & 0.905 & 0.907 & 1.097 & 1.101 & 1.062 & 7.31 \\
\hline$C 1^{f}$ & 3.646 & 429846 & 0.910 & 0.817 & 1.061 & 1.078 & 1.186 & 1.153 & 0.547 & 0.660 & 1.209 & 1.258 & 1.075 & 1.045 & 22.31 \\
\hline$C 2^{f}$ & 9.435 & 168667 & 0.994 & 0.984 & 0.986 & 0.987 & 0.925 & 0.915 & 1.140 & 1.133 & 0.938 & 0.938 & 1.026 & 1.033 & 7.39 \\
\hline$C 3^{f}$ & 9.538 & 138705 & 1.271 & 1.258 & 1.424 & 1.368 & 1.336 & 1.372 & 0.791 & 0.748 & 0.570 & 0.576 & 0.642 & 0.643 & 36.10 \\
\hline$C 4^{f}$ & 9.977 & 352654 & 0.907 & 0.885 & 0.902 & 0.894 & 0.862 & 1.138 & 1.307 & 1.034 & 0.861 & 0.877 & 1.164 & 1.169 & 15.57 \\
\hline$C 5^{f}$ & 14.846 & 152146 & 0.936 & 0.898 & 0.905 & 0.909 & 0.831 & 0.849 & 1.248 & 1.232 & 0.997 & 1.015 & 1.071 & 1.110 & 14.01 \\
\hline$C 6^{f}$ & 15.173 & 385975 & 1.097 & 1.091 & 1.076 & 1.112 & 1.011 & 1.012 & 1.123 & 1.122 & 0.821 & 0.833 & 0.848 & 0.855 & 12.44 \\
\hline$C 7^{f}$ & 15.394 & 108146 & 0.991 & 0.990 & 1.002 & 1.004 & 0.964 & 0.951 & 1.127 & 1.120 & 0.909 & 0.926 & 1.003 & 1.015 & 6.63 \\
\hline$C 8^{f}$ & 16.281 & 2457611 & 1.035 & 1.040 & 0.731 & 0.723 & 1.277 & 1.282 & 0.969 & 0.969 & 0.993 & 1.017 & 0.971 & 0.994 & 16.83 \\
\hline$C 9^{f}$ & 16.691 & 220362 & 1.243 & 1.235 & 1.312 & 1.379 & 1.584 & 1.574 & 0.763 & 0.763 & 0.444 & 0.598 & 0.545 & 0.561 & 42.74 \\
\hline $\mathrm{C} 10^{f}$ & 17.015 & 168950 & 0.878 & 0.875 & 0.605 & 0.702 & 1.136 & 1.113 & 1.126 & 1.120 & 1.146 & 1.152 & 0.997 & 1.149 & 19.17 \\
\hline $\mathrm{C} 11^{f}$ & 17.713 & 211302 & 0.783 & 0.837 & 0.782 & 0.803 & 1.109 & 1.100 & 1.062 & 1.062 & 1.111 & 1.079 & 1.015 & 1.256 & 15.78 \\
\hline $\mathrm{C} 12^{f}$ & 18.017 & 217763 & 0.859 & 0.812 & 0.819 & 0.899 & 1.021 & 1.013 & 1.079 & 1.061 & 1.105 & 1.071 & 0.996 & 1.265 & 13.32 \\
\hline
\end{tabular}

d represented the peak recorded at $254 \mathrm{~nm}$; e represented the peak recorded at $280 \mathrm{~nm}$; f represented the peak recorded at $440 \mathrm{~nm}$. 


\subsubsection{Evaluation of similarity}

Similarity evaluation software was utilized to calculate the similarity based on the "vector cosine" method[32] between different samples of ZZDHD and the control fingerprint. The control fingerprint was generated from 12 batches of ZZDHD samples (Table 6). The results showed that the similarity of single- and co-boiled ZZDHD was higher than 0.997 at $254 \mathrm{~nm}, 280 \mathrm{~nm}$, and $440 \mathrm{~nm}$, indicating that there was no significant change in chemical substances in the two different preparation methods.

Table 6

Similarity analysis of Zhi-Zi-Da-Huang decoction samples.

\begin{tabular}{|c|c|c|c|c|c|c|c|}
\hline \multirow[t]{2}{*}{ Sample No. } & \multicolumn{3}{|c|}{ Similarity } & \multirow[t]{2}{*}{ Sample No. } & \multicolumn{3}{|c|}{ Similarity } \\
\hline & $254 \mathrm{~nm}$ & $280 \mathrm{~nm}$ & $440 \mathrm{~nm}$ & & $254 \mathrm{~nm}$ & $280 \mathrm{~nm}$ & $440 \mathrm{~nm}$ \\
\hline S1 & 1.000 & 1.000 & 1.000 & S7 & 0.999 & 0.997 & 0.999 \\
\hline S2 & 0.999 & 1.000 & 1.000 & S8 & 0.999 & 0.998 & 0.999 \\
\hline S3 & 0.999 & 1.000 & 0.999 & S9 & 0.999 & 1.000 & 0.999 \\
\hline S4 & 0.999 & 1.000 & 0.999 & S10 & 0.999 & 1.000 & 0.999 \\
\hline S5 & 0.999 & 1.000 & 0.999 & S11 & 1.000 & 1.000 & 0.998 \\
\hline S6 & 0.999 & 1.000 & 0.999 & $\mathrm{~S} 12$ & 1.000 & 1.000 & 0.998 \\
\hline
\end{tabular}

\subsubsection{Chemometric analysis}

Further quality assessment of the samples was performed using a PCA on 12 samples with 30 common peaks. The results showed that there was a separation between CB and SB (Fig. 6A). These samples were clearly clustered into two groups: Group 1 including six samples (S1-S6) and Group 2 including six samples (S7-S12), which indicated that the different preparation methods resulted in changes to the ZZDHD. PCA was also employed to map the RPA of 30 common peaks onto the samples in the loading plot (Fig. 6B), which was utilized to find the compounds accountable for the separation of the groups.

Before subjecting different samples to HCA, 30 common peaks were selected. To visualize the classification trends in ZZDHD samples, HCA was conducted based on the RPA of 30 common peaks, which could divide tested samples into different categories. As the dendrogram showed in Fig. $6 \mathrm{C}, 12$ batches of ZZDHD samples were sorted into two clusters. All CB samples (S1-S6) were grouped as group I, and SB samples (S7-S12) were grouped as group II. This corresponded to the PCA plot shown in Fig. 6A, although the similarity values of these samples were much closer. It was concluded that the classification of the sample quality might be mostly relevant to the boiling method of ZZDHD.

Based on the results of the HCA, PLS-DA was further established to obtain important variables with CB and SB samples. The VIP plot displays the importance of variables both to explain X correlated to Y. Based on the VIP values (i.e., larger than 1.0), 13 compounds played key roles in the clustering (Fig. 7). These 13 compounds, including C3, A3, crocetin, daidzin, C5, physcion, G2, G1, chrysophanol, emodin, A2, A5, and C6, corresponded to those points far away from zero in the loading plot of 30 common peaks (Fig. 6B).

\section{Discussion}

Currently, Traditional Chinese Medicine decoctions and formula granules have been widely studied and applied around the world. The study of chemical compounds is the key factor for the development of traditional Chinese medicine decoctions into formula granules. Unambiguous chemical substances guarantees the quality and therapeutic efficacy of Traditional Chinese Medicine decoctions, so investigations of Traditional Chinese Medicine is essential. However, the chemical composition of Traditional Chinese Medicines is rather complicated, which poses great challenge for the separation and identification of their chemical compositions. UPLC-qTOF-MS/MS has allowed for the perfect combination of ultra-high performance liquid chromatography and highquality mass spectrometry with high resolution, which have improved performances compared with LC-MS. UPLC uses small particle packing (1.7 $\mu$ m) as the stationary phase to achieve faster, more sensitive, and higher resolution chromatographic separation of samples, which has advantages over traditional HPLC. QTOF-MS/MS with high quality resolution and high sensitivity can accurately determine molecular weight and predict the molecular composition[33]. UPLCqTOF-MS/MS can be used for qualitative or quantitative analysis of trace samples with extremely complex components, especially for the study of metabonomics and drug metabolism. Therefore, UPLC-qTOF-MS/MS was used to separate and analyze the chemical components in ZZDHD. A total of 147 chemical compounds were preliminarily determined, and 11 compounds found in CB were distinguishing components of the single- and co-boiled ZZDHD. Our results indicated that chemical compounds changed after the combination of four herbs. Through comparing the mass spectrometry information of the single-boiled herbs, affiliations of 11 compounds were clarified. Gallic acid-4-O-ß-D-glucopyranoside, glucosylsyringic acid, Unknown 4, gallate-O-diglucoside, and glycerinmonogallete were derived from DH. Unknown 15 originated from ZZ. In addition, 3', 4', 5, 5', 6, and 7-Hexamethoxyflavone was present in ZS. Importantly, Unknown 59, Unknown 61, Unknown 66, and Unknown 69 were not found in the four herbs or SB. We speculated that the co-boiling of the four herbs together might result in detection of the former six compounds and the generation of the other four compounds. However, there are no further study on the specific mechanism. 
Previous studies showed that iridoids, anthraquinones, flavonoids, isoflavonoids, coumarins, and crocetin glycosides were the major bioactive compounds in ZZDHD[28]. Based on this, we determined 15 representative compounds by UPLC-DAD in order to further evaluate the quality of samples and comprehensively compare the differences of chemical substances in ZZDHD. ZZDHD has been extensively used for clearing heat and eliminating dampness and jaundice for many years in China, and it is further thought to have liver-protective, choleretic, and anti-inflammatory effects. A previous study has reported that geniposide could prevent hepatocyte injury[34]. Geniposide is hydrolyzed into genipin under the action of beta-D-glucosidases in intestinal bacteria, which is required for its choleretic action[35]. Genipin could also influence the induction of the inflammatory mediators inducible nitric oxide synthase (iNOS) and tumor necrosis factor (TNF)-a through the inhibition of nuclear transcription factor-kB (NF-kB) activation in hepatocytes; therefore, it might have therapeutic potential for liver injury[36]. Moreover, as one of the major pharmacodynamic substances, G1 generated geniposide and genipin via deglycosylation involving intestinal bacteria, and then distributed in the target organ for hepatinica and cholagogue[37]. As one of the primary constituents of crocin, Crocin-I could reduce oxidative damage in liver tissue in diabetic rats[38]. Crocetin exhibited inhibitory potential against cyclooxygenase-2 (COX-2), iNOS, proinflammatory factors (interleukin [IL]-1 $\beta$, IL-6, IL-10, and TNF-a) in in vitro assays, and it significantly decreased hepatic parameters, such as serum glutamic-oxaloacetic transaminase (SGOT), serum glutamic pyruvic transaminase (SGPT), and alkaline phosphatase (ALP), which suggested protective and therapeutic effects on inflammatory tissue[39]. DH and its anthraquinone constituents, including rhein, emodin, and chrysophanol, could exert a protective effect against liver injury[18, 40-42]. Emerging studies suggested that physcion suppressed hepatocellular carcinoma cell metastasis[43]. Narirutin, naringin, hesperidin, and neohesperidin, the active constituents of ZS, could inhibit pro-inflammatory factors, attenuate liver damage, modulate the state of antioxidants, and have hypoglycemic and hypolipidemic effects[44-47]. Some studies have revealed that the major isoflavonoids of DDC, such as daidzein, have a significant hepatoprotective effect against oxidative damage induced by tert-butylhydroperoxide (TBHP) in rat hepatocyte BRL-3A cell line, which is closely related to the efficacy of ZZDHD $15,16,18]$. Some pharmacokinetics indicated that isoflavone glycosides of DDC, including daidzein and genistin, could be hydrolyzed in the intestinal wall by bacterial $\beta$-glucosidases after oral administration, and further transformed into corresponding bioactive aglycones, and all of them could be excreted into bile[48]. As a glucoside form of isoflavones, daidzin markedly reduced the elevated serum aminotransferase activity and attenuated the apoptosis of hepatocytes[49]. Another study reported that the complex interactions among four herbs may result in a synergistic or antagonistic effect on the solubility of active compounds[50]. Therefore, it was reasonable to select these quantitative compounds, and all of 15 quantitative compounds were representative compounds in four herbs as well.

Then we used fingerprinting to evaluate the similarity of 30 common peaks in 12 batches of samples. The number and retention time of peaks in CB were similar to that of SB, but the peak area of many peaks increased. We therefore conclude that co-boiling was beneficial to the dissolution of effective herbal compounds, providing scientific support for traditional decoction preparation. Determination and chemometric analysis of the fingerprinting were integrated. In 30 common peaks, we found that crocetin, daidzin, physcion, G2, chrysophanol, and emodin with contents changed were included in 15 quantitative compounds. The change in content might be one of the reasons responsible for the discrimination of CB and SB; therefore, these compounds may be effective quality control markers to differentiate the two methods of preparation of ZZDHD. To our knowledge, the quality standard of ZZDHD has not yet been described by the Pharmacopoeia of the People's Republic of China. The quantification, fingerprinting methods, and results of the 15 compounds presented in this study could provide a reference for the establishment of quality control of ZZDHD.

\section{Conclusion}

In the present research, a rapid and efficient method to integrate UPLC with UPLC-qTOF-MS/MS analysis was successfully established to comprehensively discriminate and assess the quality of ZZDHD samples. Simultaneously, combining chemometric analysis with chromatographic fingerprints served as a powerful tool to apply to the quality control of the co- and single-boiled ZZDHD. We identified a total of 147 chemical compounds, 15 of which were quantified and 12 batches of samples were qualitatively fingerprinted for a systematic analysis of the chemical substances of the two preparation methods of ZZDHD. This study shows the difference of chemical substances between two preparation methods of ZZDHD, which provided an experimental basis for explaining the rationality of the preparation methods. However, research remains limited on the pharmacodynamics, clinical verification, and correlation between changes in chemical composition, pharmacodynamics, and clinical efficacy of ZZDHD prepared via different methods; therefore, further research is required.

\section{Abbreviations}

ZZDHD: Zhi-Zi-Da-Huang decoction; ZZ: Gardeniae Fructus; DH: Rhei Radix et Rhizoma; ZS: Fructus Aurantii Immaturus; DDC: Sojae Semen Praeparatum; CB: co-boiled Zhi-Zi-Da-Huang decoction; SB: single-boiled Zhi-Zi-Da-Huang decoction; MS: mass spectrometry; UHPLC: ultra-high-performance liquid chromatography; UPLC-qTOF-MS/MS: ultra-performance liquid chromatography-quadrupole time-of-flight mass spectrometry; PCA: principal component analysis; PLS-DA: partial least-squares discrimination analysis; HCA: hierarchical cluster analysis; G1: genipin-1-0- $\beta$-D-gentiobioside; G2: geniposide; RPA: relative peak area; VIP: variable importance in projection; RSD: relative standard diviation; RRT: relative retention time; TNF: tumor necrosis factor; iNOS: inducible nitric oxide synthase; TNF: tumor necrosis factor; NF-KB: nuclear transcription factor-kB; COX-2: cyclooxygenase-2; SGOT: serum glutamicoxaloacetic transaminase; SGPT: serum glutamic pyruvic transaminase; ALP: alkaline phosphatase; TBHP: tert-butylhydroperoxide.

\section{Declarations}

\section{Acknowledgements}

Not applicable.

\section{Authors'contributions}


$\mathrm{CZ}, \mathrm{XZ}$ and $\mathrm{YW}$ were responsible for the design of this study. $\mathrm{XZ}$ and $\mathrm{HW}$ collected and detected samples. MX and HW analyed data. MX, XZ and CZ writed and revised the article. All authors read and approved the final manuscript.

\section{Funding}

The financial support from Fundamental Research Funds for the Central public welfare research institutes of China Academy of Chinese Medical Sciences (ZZ13-019, ZXKT17044), the National Natural Science Foundation of China (81873010, 81703708) and project of Beijing for traditional Chinese Medicine Processing Technology inheritance base are gratefully acknowledged.

\section{Availability of data and materials}

Not applicable.

\section{Ethics approval and consent to participate}

Not applicable.

\section{Consent for publication}

Not applicable.

\section{Competing interests}

The authors declare that they have no competing interests.

\section{Author details}

${ }^{1}$ Institute of Chinese Materia Medica, China Academy of Chinese Medical Sciences, Beijing 100700, China. ${ }^{2}$ College of Pharmacy, Henan University of Chinese Medicine, Zhengzhou 450008, China. ${ }^{3}$ China Resources Sanjiu Medical \&Pharmaceutical Co., Ltd., Shenzhen 518000, China.

\section{References}

1. Yang B, Liu Z, Wang Q, Xia P. Chemical interaction between Lilium brownii and Rhizoma Anemarrhenae, the herbal constituents of Baihe Zhimu decoction, by liquid chromatography coupled to hybrid triple quadrupole linear ion trap mass spectrometer. Biomed Chromatogr. 2018; 32(3):10.1002/bmc.4118.

2. Tian Q, Liu F, Xu Z, Liu H, Yin H, Sun Z, et al. Evaluation of the chemical consistency of Yin-Chen-Hao-Tang prepared by combined and separated decoction methods using high-performance liquid chromatography and quadrupole time-of-flight mass spectrometry coupled with multivariate statistical analysis. J Sep Sci. 2019;42(9):1664-75.

3. Liang G, Cao P, Chai L, Xian F, Li X. Effects of different compounding of formulae on content of gardenoside in Yin Chen Hao decoction. J Tradit Chin Med. 2002;22(1):55-60.

4. Yingying Z, Qiaoyu J, Qing L. Research status of prescription granules of traditional Chinese medicine. Asia-Pacific Traditional Medicine. 2018;14(12):946.

5. Li R, Zhai H-Q, Tian W-L, Hou J-R, Jin S-Y, Wang Y-Y. Comparative analysis between origin of cooked traditional Chinese medicine powder and modern formula granules. Zhongguo Zhong yao za zhi. 2016;41(5):965-9.

6. Yongyan W, Qingwei L, Hongliang L. Reflections on traditional Chinese medicine for formula granules and current problems. Central South Pharmacy. 2019;17(01):78-83.

7. Wu C, Zhang J, Zhao Y, Chen J, Liu Y. Chinese herbal medicine bushen qinggan formula for blood pressure variability and endothelial injury in hypertensive patients: a randomized controlled pilot clinical trial. Evid Based Complement Alternat Med. 2014; 2014:804171-804171.

8. Li S-L, Song J-Z, Qiao C-F, Zhou Y, Xu H-X. UPLC-PDA-TOFMS based chemical profiling approach to rapidly evaluate chemical consistency between traditional and dispensing granule decoctions of traditional medicine combinatorial formulae. J Pharm Biomed Anal. 2010;52(4):468-78.

9. Liu J, Zhang Y, Hao Y, Zhao Y, Li Y, Qin R, et al. Chemical composition differentiation of Shen-Shuai-Ning granule between combined decoction and separated decoction using HPLC-DAD-ESI-QTOF-MS. Biomed Chromatogr. 2017; 31(9):10.1002/bmc.3949.

10. Ma C, Qian Y, Fan X, Shang E, Yao X, Ma S. Using UPLC-QTOF-MS to analyze the chemical changes between traditional and dispensing granule decoctions of San-Ao-Tang. J Chromatogr Sci. 2014;52(4):277-92.

11. Shang E, Zhu Z, Liu L, Tang Y, Duan J-A. UPLC-QTOF-MS with chemical profiling approach for rapidly evaluating chemical consistency between traditional and dispensing granule decoctions of Tao-Hong-Si-Wu decoction. Chem Cent J. 2012;6(1):143-3.

12. Xin Z, Shuo W, Wensheng Q. Clinical observation on 80 cases of coronary heart disease angina pectoris with heat stagnation and blood stasis syndrome treated by modified Zhi-Zi Da-Huang decoction. Beijing Journal of Traditional Chinese Medicine. 2010;29(10):775-6+806.

13. An L, Lang Q, Shen W, Shi Q, Feng F. Dynamic metabolic profiling of urine biomarkers in rats with alcoholinduced liver damage following treatment with ZhiZiDaHuang decoction. Mol Med Rep. 2016;14(3):2093-100.

14. Chen KL, Bi KS, Han F, Zhu HY, Zhang XS, Mao XJ, et al. Evaluation of the protective effect of Zhi-Zi-da-Huang decoction on acute liver injury with cholestasis induced by alpha-naphthylisothiocyanate in rats. J Ethnopharmacol. 2015;172:402-9. 
15. Zhu H, Bi K, Han F, Guan J, Tang Z, Chen K, et al. Simultaneous determination of two iridoid glycosides, two anthraquinones and four flavonoid glycosides of Zhi-Zi-Da-Huang decoction in rat plasma by UFLC-MS/MS: application to a comparative pharmacokinetic study in normal and cholestatic liver injury rats. J Chromatogr B Analyt Technol Biomed Life Sci. 2014;960:116-25.

16. Wang S, Li X, Niu Y, Liu Y, Zhu Y, Lu X, et al. Identification and screening of chemical constituents with hepatoprotective effects from three traditional Chinese medicines for treating jaundice. J Sep Sci. 2016;39(19):3690-9.

17. Wenjia W, Shumin W, Ya D. Clinical observation of Zhi-Zi Da-Huang decoction combined with Chai-Hu Shu-Gan powder in treating jaundice hepatitis. Chin J Tradit Med Sci Technol. 2019;26(04):593-5.

18. Wang H, Feng F, Zhuang BY, Sun Y. Evaluation of hepatoprotective effect of Zhi-Zi-Da-Huang decoction and its two fractions against acute alcoholinduced liver injury in rats. J Ethnopharmacol. 2009;126(2):273-9.

19. Zhan C, Xiong A, Shen D, Yang L, Wang Z. Characterization of the Principal Constituents of Danning Tablets, a Chinese Formula Consisting of Seven Herbs, by an UPLC-DAD-MS/MS Approach. Molecules. 2016;21(5):631.

20. Qing H, Xixi L, Qingshui S, Gongjun Y, Fang F. Deciphering the absorption profile and interaction of multi-components of Zhi-Zi-Da-Huang decoction based on in vitro-in silico-in vivo integrated strategy. Xenobiotica. 2019; 49(7).

21. Li HB, Yu Y, Wang ZZ, Dai Y, Gao H, Xiao W, et al. Iridoid and bis-iridoid glucosides from the fruit of Gardenia jasminoides. Fitoterapia. $2013 ; 88: 7-11$.

22. Feng J, He X, Zhou S, Peng F, Liu J, Hao L, et al. Preparative separation of crocins and geniposide simultaneously from gardenia fruits using macroporous resin and reversed-phase chromatography. J Sep Sci. 2014;37(3):314-22.

23. Wang C, Pan Y, Fan G, Chai Y, Wu Y. Application of an efficient strategy based on MAE, HPLC-DAD-MS/MS and HSCCC for the rapid extraction, identification, separation and purification of flavonoids from Fructus Aurantii Immaturus. Biomed Chromatogr. 2010;24(3):235-44.

24. Qu L-p, Fan G-r, Peng J-y. Mi H-m. Isolation of six isoflavones from Semen sojae praeparatum by preparative HPLC. Fitoterapia. 2007;78(3):200-4.

25. Qin Y, Zhou R, Huang J, Jin J, Zhou Q, Liu H, et al. Comprehensive comparison of the anti-inflammatory activity and chemical consistency of traditional Chinese medicine formula granules with Ge-Gen decoction as a representative sample. Biomed Chromatogr. 2019;33(12):e4689-9.

26. Hai-Qiang W, Yun-Xiang Z, Yi-Ning L, Ruo-Liu W, Shu-Fang W. Rapid discovery and identification of the anti-inflammatory constituents in Zhi-Shi-Zhi-ZiChi-Tang. Chin J Nat Med. 2019;17(4):308-20.

27. Wu X, Zhou Y, Yin F, Mao C, Li L, Cai B, et al. Quality control and producing areas differentiation of Gardeniae Fructus for eight bioactive constituents by HPLC-DAD-ESI/MS. Phytomedicine. 2014;21(4):551-9.

28. Zhu H, Yin R, Han F, Guan J, Zhang X, Mao X, et al. Characterization of chemical constituents in Zhi-Zi-Da-Huang decoction by ultra high performance liquid chromatography coupled with quadrupole time-of-flight mass spectrometry. J Sep Sci. 2014;37(23):3489-96.

29. Wang H, Feng F. Identification of components in Zhi-Zi-Da-Huang decoction by HPLC coupled with electrospray ionization tandem mass spectrometry, photodiode array and fluorescence detectors. J Pharm Biomed Anal. 2009;49(5):1157-65.

30. Sheu SJ, Hsin WC. HPLC separation of the major constituents of Gardeniae fructus. J High Resolut Chromatogr. 1998;21(9):523-6.

31. Chai C, Cui X, Shan C, Yu S, Wang X, Wen H. Simultaneous Characterization and Quantification of Varied Ingredients from Sojae semen praeparatum in Fermentation Using UFLCâđ»TripleTOF MS. Molecules. 2019;24(10):1864.

32. Liu Y, Meng Q, Jiang S, Hu Y. [Similarity system theory to evaluate similarity of chromatographic fingerprints of traditional Chinese medicine]. Se Pu. 2005;23(2):158-63.

33. Ibáñez C, García-Cañas V, Valdés A, Simó C. Novel MS-based approaches and applications in food metabolomics. TrAC Trends Anal Chem. 2013;52:10011.

34. Shen B, Feng H, Cheng J, Li Z, Jin M, Zhao L, et al. Geniposide alleviates non-alcohol fatty liver disease via regulating Nrf2/AMPK/mTOR signalling pathways. J Cell Mol Med. 2020;24(9):5097-108.

35. Akao T, Kobashi K, Aburada M. Enzymic studies on the animal and intestinal bacterial metabolism of geniposide. Biol Pharm Bull. 1994;17(12):1573-6.

36. Nakatake R, Tsuda T, Matsuura T, Miki H, Hishikawa H, Matsushima H, et al. Genipin Inhibits the Induction of Inducible Nitric Oxide Synthase Through the Inhibition of NF-KB Activation in Rat Hepatocytes. Drug Metab Lett. 2017;10(4):254-63.

37. Jiang P, Ma Y, Gao Y, Li Z, Lian S, Xu Z, et al. Comprehensive Evaluation of the Metabolism of Genipin-1- $\beta$-d-gentiobioside in Vitro and in Vivo by Using HPLC-Q-TOF. J Agric Food Chem. 2016;64(27):5490-8.

38. Yaribeygi H, Mohammadi MT, Sahebkar A. Crocin potentiates antioxidant defense system and improves oxidative damage in liver tissue in diabetic rats. Biomed Pharmacother. 2018;98:333-7.

39. Li Y, Kakkar R, Wang J. In vivo and in vitro Approach to Anti-arthritic and Anti-inflammatory Effect of Crocetin by Alteration of Nuclear Factor-E2-Related Factor 2/hem Oxygenase (HO)-1 and NF-KB Expression. Front Pharmacol. 2018;9:1341.

40. Xing XY, Zhao YL, Kong WJ, Wang JB, Jia L, Zhang P, et al. Investigation of the "dose-time-response" relationships of rhubarb on carbon tetrachlorideinduced liver injury in rats. J Ethnopharmacol. 2011;135(2):575-81.

41. Yang F, Xu Y, Xiong A, He Y, Yang L, Wan YJ, et al. Evaluation of the protective effect of Rhei Radix et Rhizoma against a-naphthylisothiocyanate induced liver injury based on metabolic profile of bile acids. J Ethnopharmacol. 2012;144(3):599-604.

42. Wang X, Sun H, Zhang A, Jiao G, Sun W, Yuan Y. Pharmacokinetics screening for multi-components absorbed in the rat plasma after oral administration traditional Chinese medicine formula Yin-Chen-Hao-Tang by ultra performance liquid chromatography-electrospray ionization/quadrupole-time-of-flight mass spectrometry combined with pattern recognition methods. Analyst. 2011;136(23):5068-76. 
43. Pan XP, Wang C, Li Y, Huang LH. Physcion induces apoptosis through triggering endoplasmic reticulum stress in hepatocellular carcinoma. Biomed Pharmacother. 2018;99:894-903.

44. Ha SK, Park HY, Eom H, Kim Y, Choi I. Narirutin fraction from citrus peels attenuates LPS-stimulated inflammatory response through inhibition of NF-KB and MAPKs activation. Food Chem Toxicol. 2012;50(10):3498-504.

45. Rodríguez V, Plavnik L, Tolosa de Talamoni N. Naringin attenuates liver damage in streptozotocin-induced diabetic rats. Biomed Pharmacother. 2018;105:95-102.

46. Martínez-Noguera FJ, Marín-Pagán C, Carlos-Vivas J, Rubio-Arias JA, Alcaraz PE. Acute Effects of Hesperidin in Oxidant/Antioxidant State Markers and Performance in Amateur Cyclists. Nutrients. 2019; 11(8).

47. Jia S, Hu Y, Zhang W, Zhao X, Chen Y, Sun C, et al. Hypoglycemic and hypolipidemic effects of neohesperidin derived from Citrus aurantium L. in diabetic KK-A(y) mice. Food Funct. 2015;6(3):878-86.

48. Yao Y, Ma X, Li T, Guo H, Chang R, Liu J, et al. Quantification of isoflavone glycosides and aglycones in rat plasma by LC-MS/MS: Troubleshooting of interference from food and its application to pharmacokinetic study of Semen Sojae Praeparatum extract. J Pharm Biomed Anal. 2018;161:444-54.

49. Kim SH, Heo JH, Kim YS, Kang SS, Choi JS, Lee SM. Protective effect of daidzin against D-galactosamine and lipopolysaccharide-induced hepatic failure in mice. Phytother Res. 2009;23(5):701-6.

50. Tang Z, Yin R, Bi K, Zhu H, Han F, Chen K, et al. Simultaneous quantitative determination of 20 active components in the traditional Chinese medicine formula Zhi-Zi-Da-Huang decoction by liquid chromatography coupled with mass spectrometry: application to study the chemical composition variations in different combinations. Biomed Chromatogr. 2015;29(9):1406-14.

\section{Figures}
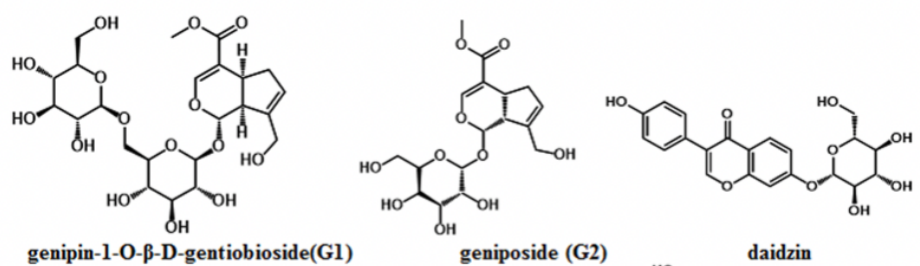

genipin-1-O- $\beta$-D-gentiobioside(Gl)
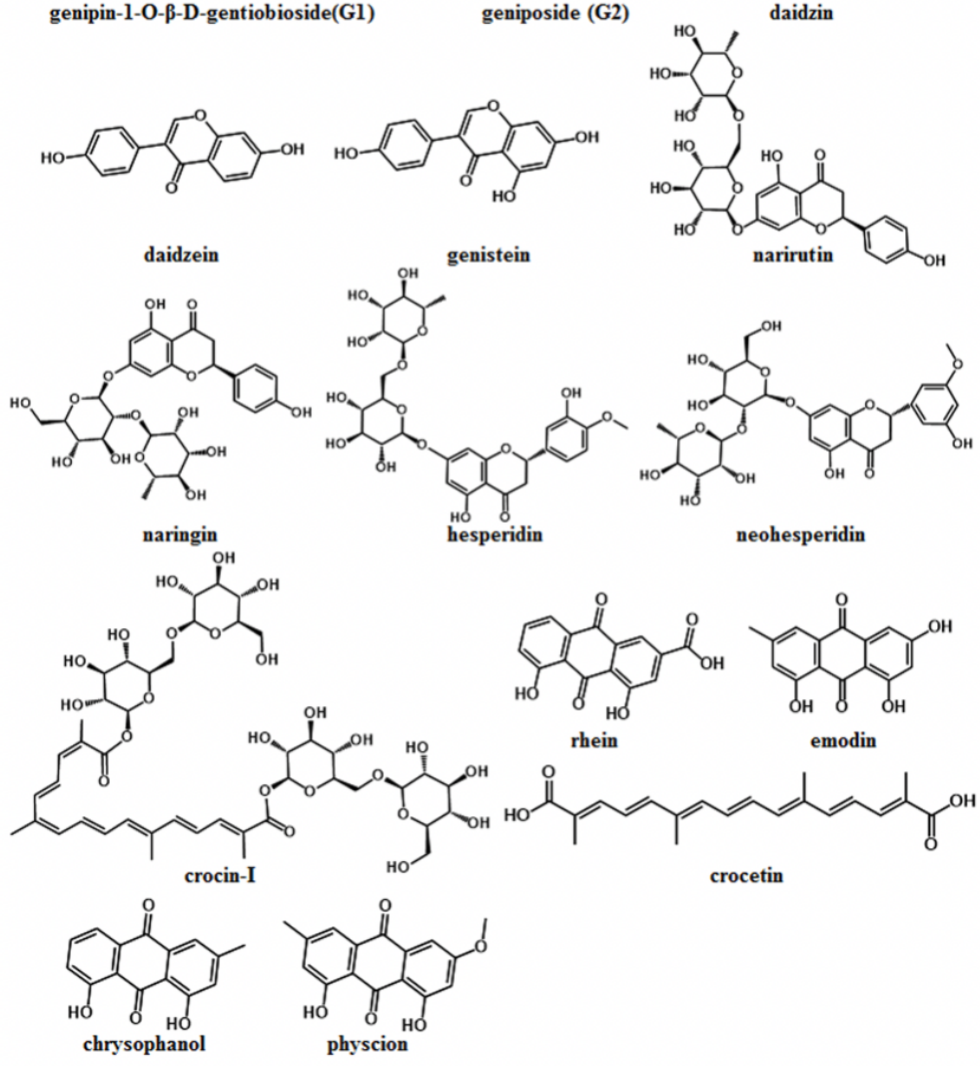

\section{Figure 1}

Chemical structures of 15 reference standards in Zhi-Zi-Da-Huang decoction. 


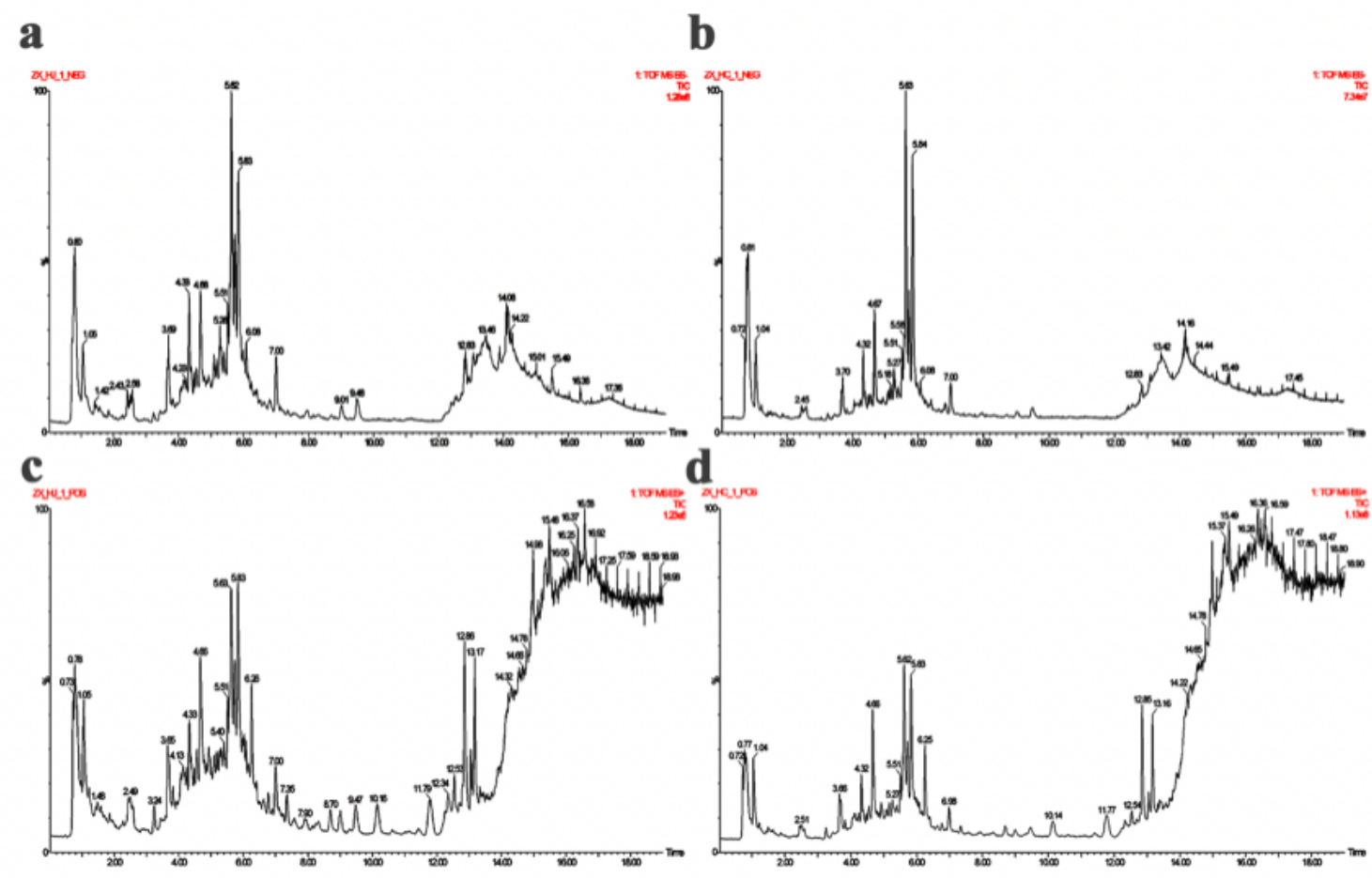

Figure 2

Total ion chromatogram of Zhi-Zi-Da-Huang decoction obtained by UPLC-qTOF-MS/MS. (a) the co-boiled Zhi-Zi-Da-Huang decoction in negative ion mode; (b) the single-boiled Zhi-Zi-Da-Huang decoction in negative ion mode; (c) the co-boiled Zhi-Zi-Da-Huang decoction in positive ion mode; (d) the single-boiled Zhi-ZiDa-Huang decoction in positive ion mode. 


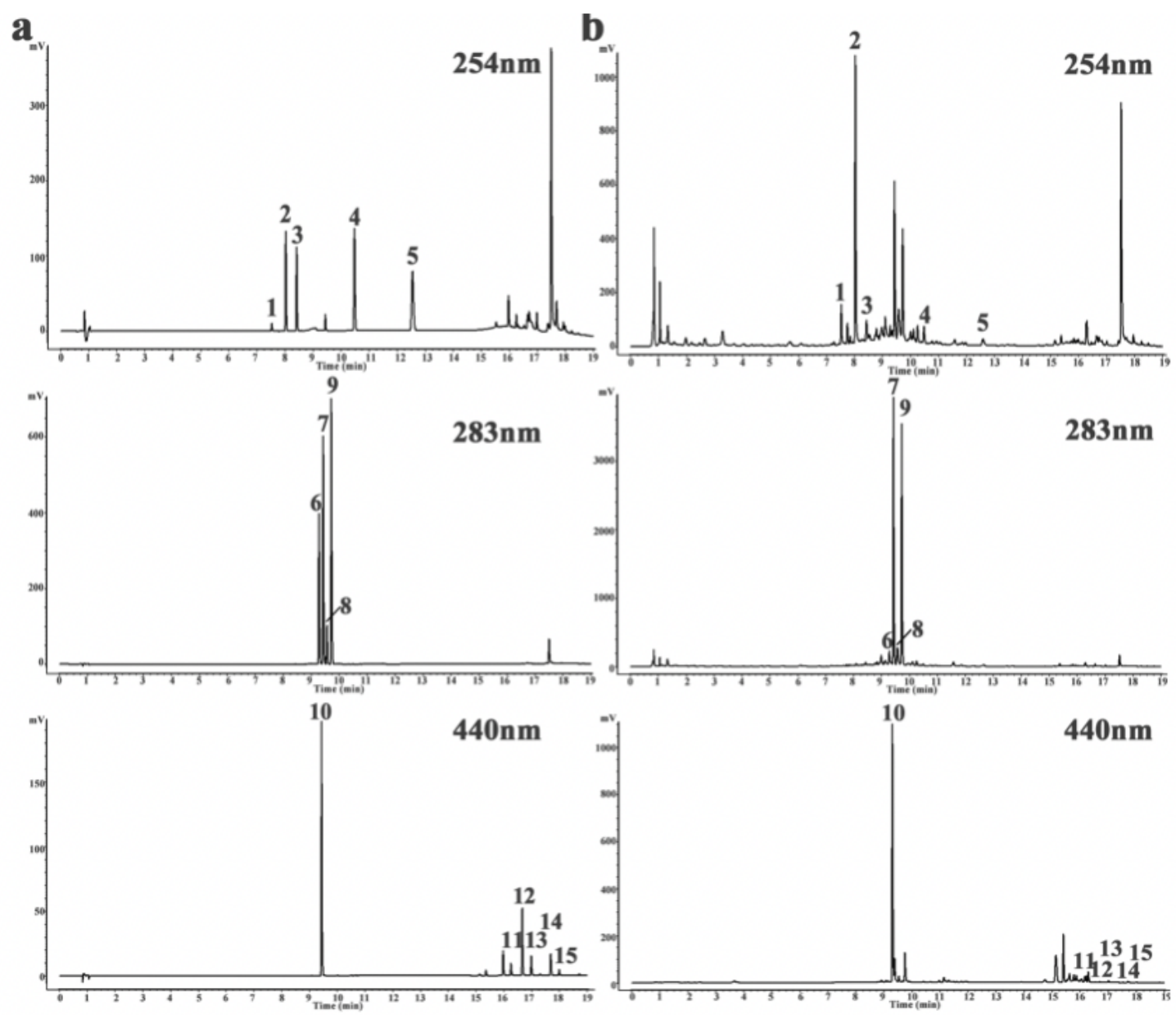

Figure 3

Representative UPLC chromatograms under $254 \mathrm{~nm}, 283 \mathrm{~nm}$ and $440 \mathrm{~nm}$. (a) the reference standards; (b) the sample. Peaks 1 to 15 were identified as genipin1-O-ß-D-gentiobioside (G1), geniposide (G2), daidzin, daidzein, genistein, narirutin, naringin, hesperidin, neohesperidin, crocin-I, rhein, crocetin, emodin, chrysophanol and physcion, respectively. (X-axi: running time; $\mathrm{Y}$-axi: peak intensity). 

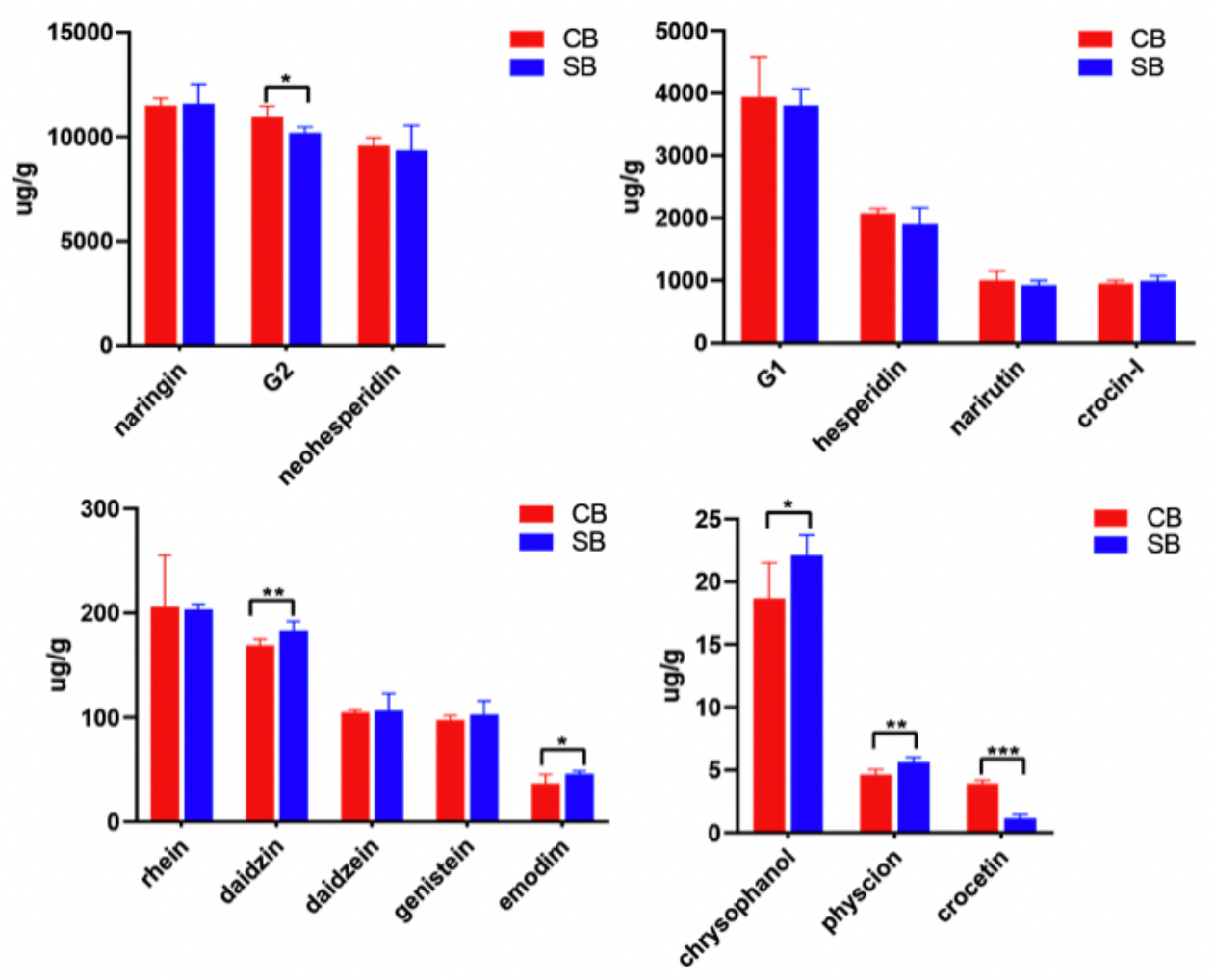

Figure 4

Comparison the content of 15 compounds of the co-boiled Zhi-Zi-Da-Huang decoction (CB) and the single-boiled Zhi-Zi-Da-Huang decoction (SB). 

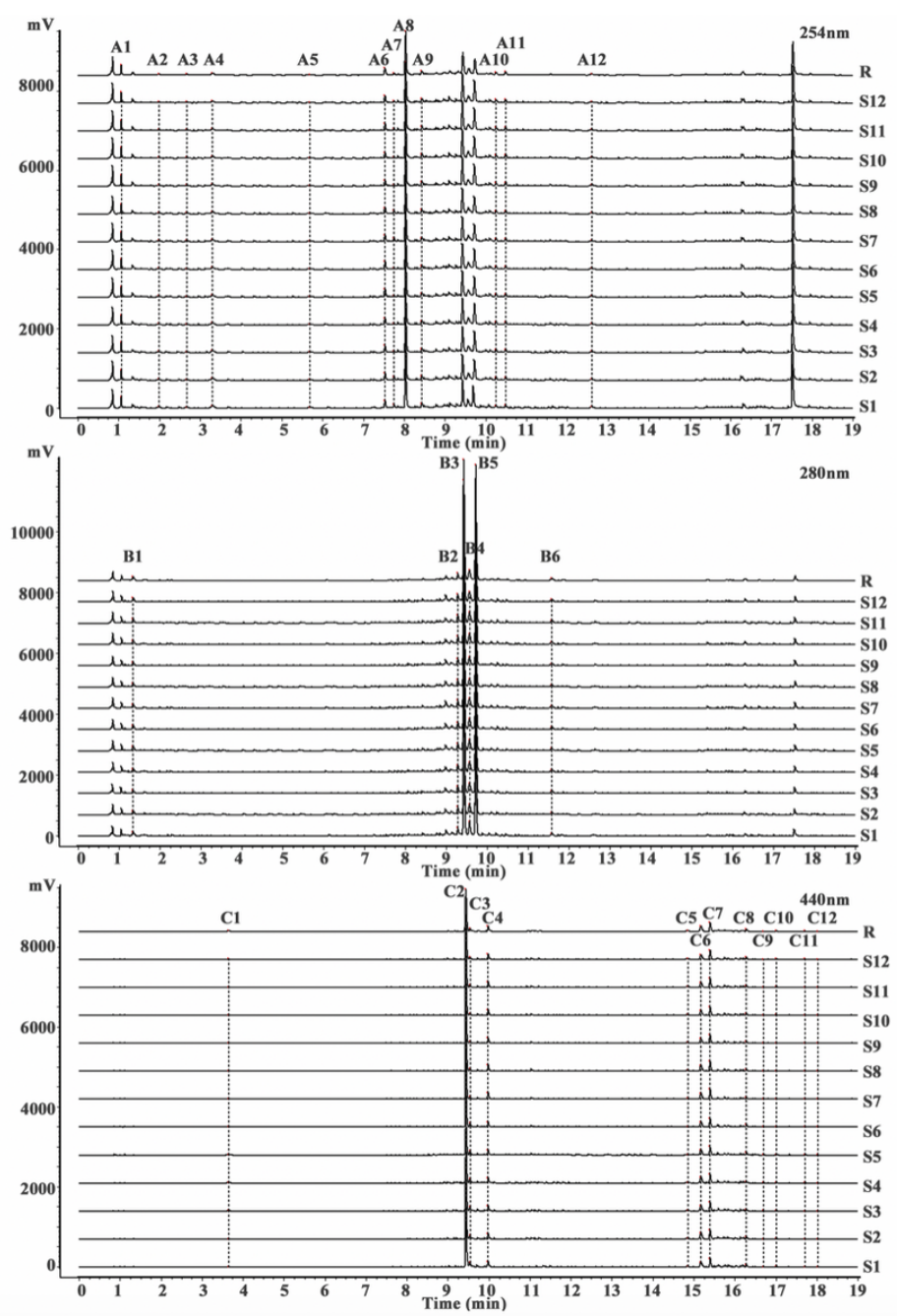

\section{Figure 5}

UPLC overlap of the co-boiled Zhi-Zi-Da-Huang decoction (S1-S6), the single-boiled Zhi-Zi-Da-Huang decoction (S7-S12) and the representative fingerprint (R). The peaks marked with $\mathrm{A} 1-\mathrm{A} 12, \mathrm{~B} 1-\mathrm{B} 6$ and $\mathrm{C} 1-\mathrm{C} 12$ represent the 30 characteristic common peaks.

a

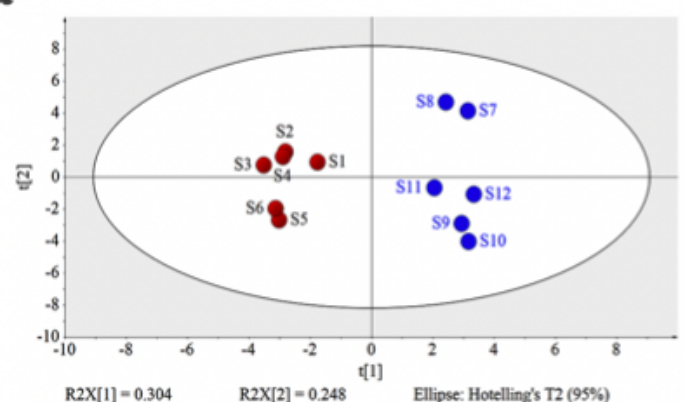

b

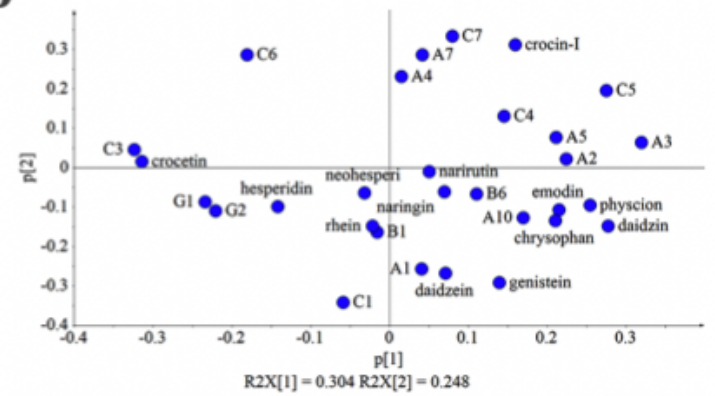

\section{c}

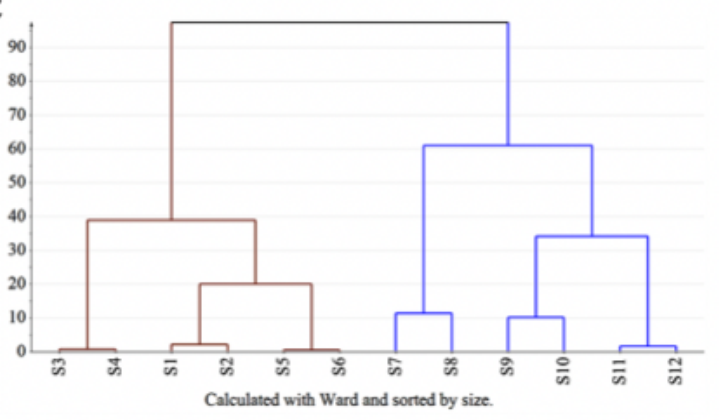


Figure 6

a, Principal component analysis plot of 12 samples of the Zhi-Zi-Da-Huang decoction. b, Loading plot of 30 common peaks. c, Hierarchical cluster analysis dendrogram of different samples of the co-boiled Zhi-Zi-Da-Huang decoction (S1-S6) and the single-boiled Zhi-Zi-Da-Huang decoction (S7-S12).

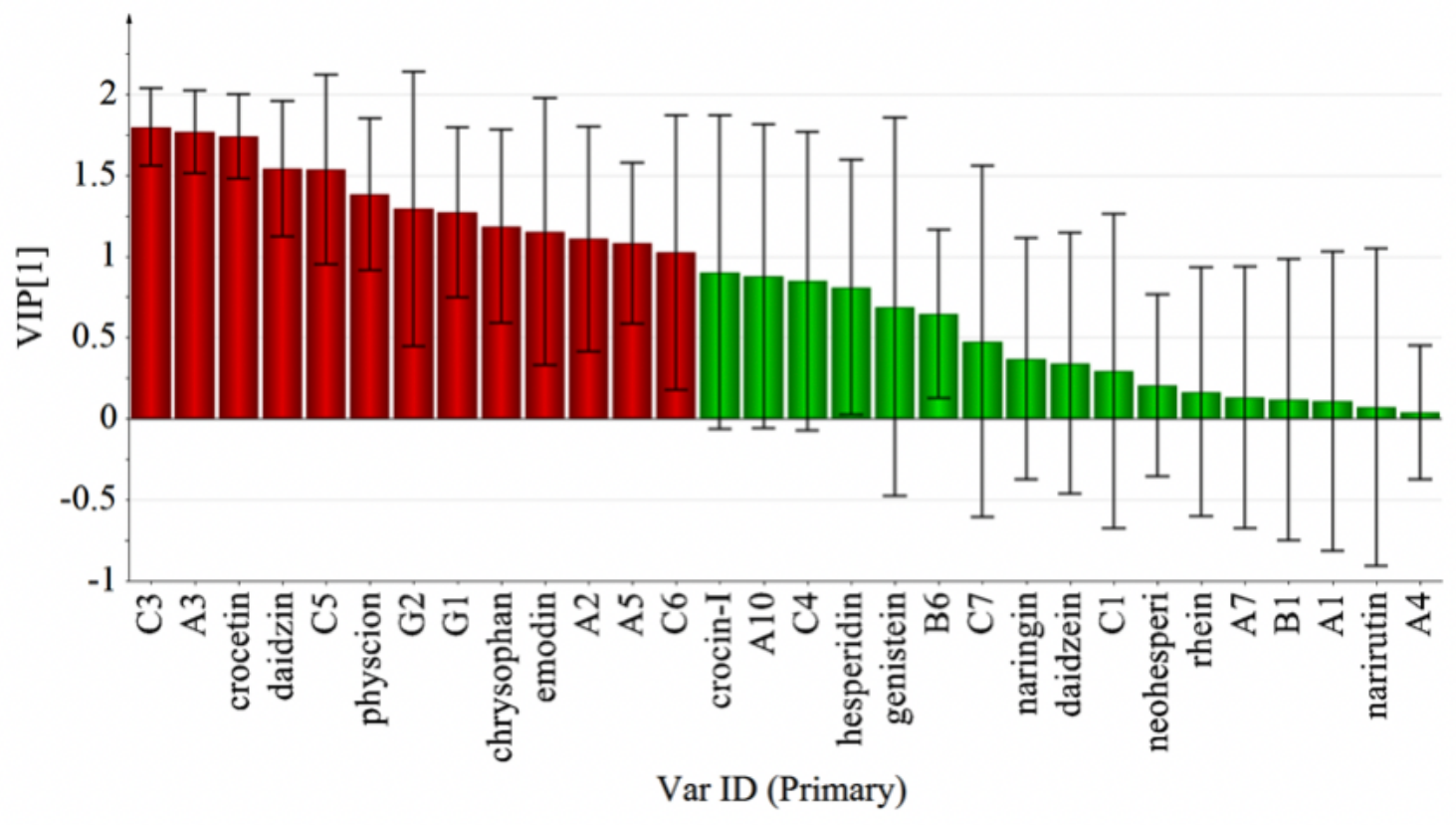

Figure 7

Variable importance in projection (VIP) plot of the co-boiled Zhi-Zi-Da-Huang decoction (CB) and the single-boiled Zhi-Zi-Da-Huang decoction (SB) with confidence intervals.

$\mathbf{a}$

geniposide<smiles>COC(=O)C1=COC(OC2OC(CO)C(O)C(O)C2O)C2C(CO)=CCC12</smiles><smiles>COC(=O)C1=COC([18OH])C2C(CO)=CCC12</smiles>

b

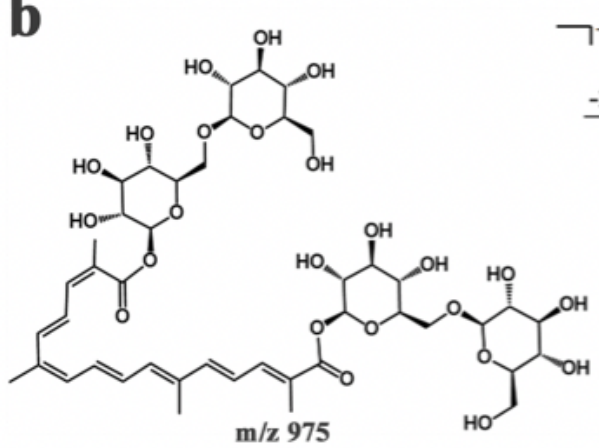

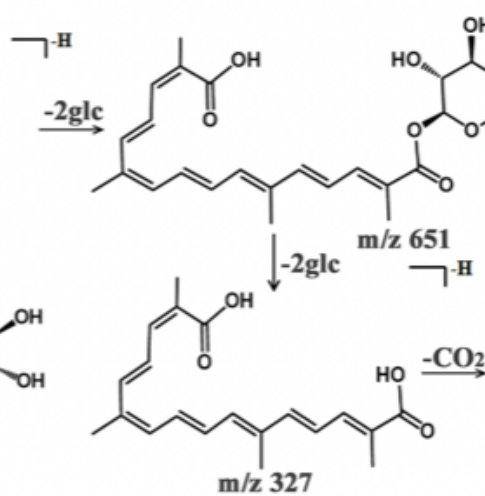<smiles>C/C=C\C=C\C(=C/C=C/C=C\C=C/C=C(/C)C(=O)O)COC1OC(OCC(C)C(C)O)C(O)C(O)C1O</smiles>

Figure 8

Fig. A1 The proposed fragmentation pathways of geniposide(a) and crocin-l(b) in the negative ion mode. 3.1.2 Identification of compounds in Fructus Aurantii Immaturus 


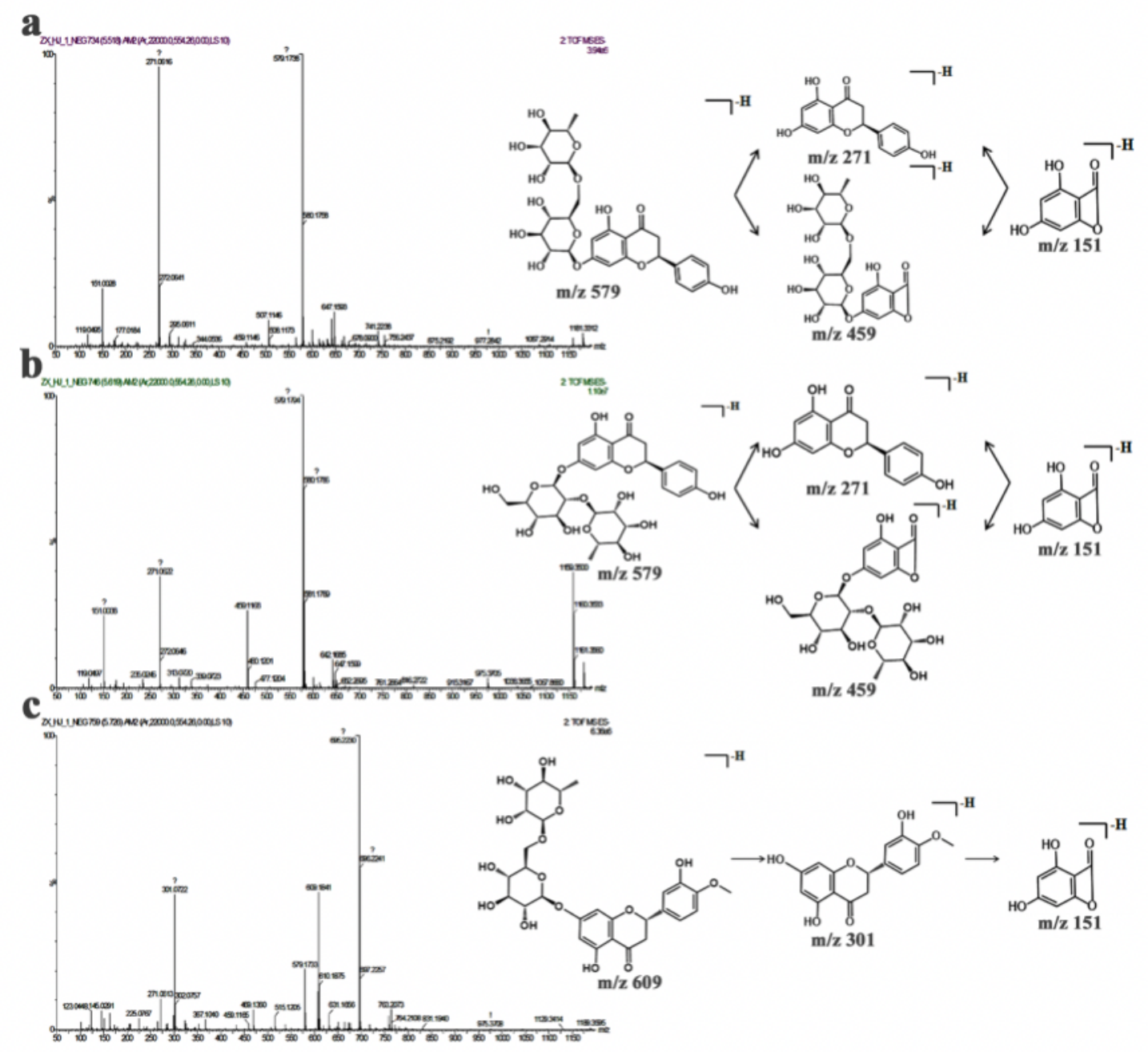

Figure 9

Fig. A2 The MS/MS spectra and proposed fragmentation pathways of narirutin(a), naringin(b) and hesperidin(c) in the negative ion mode. 


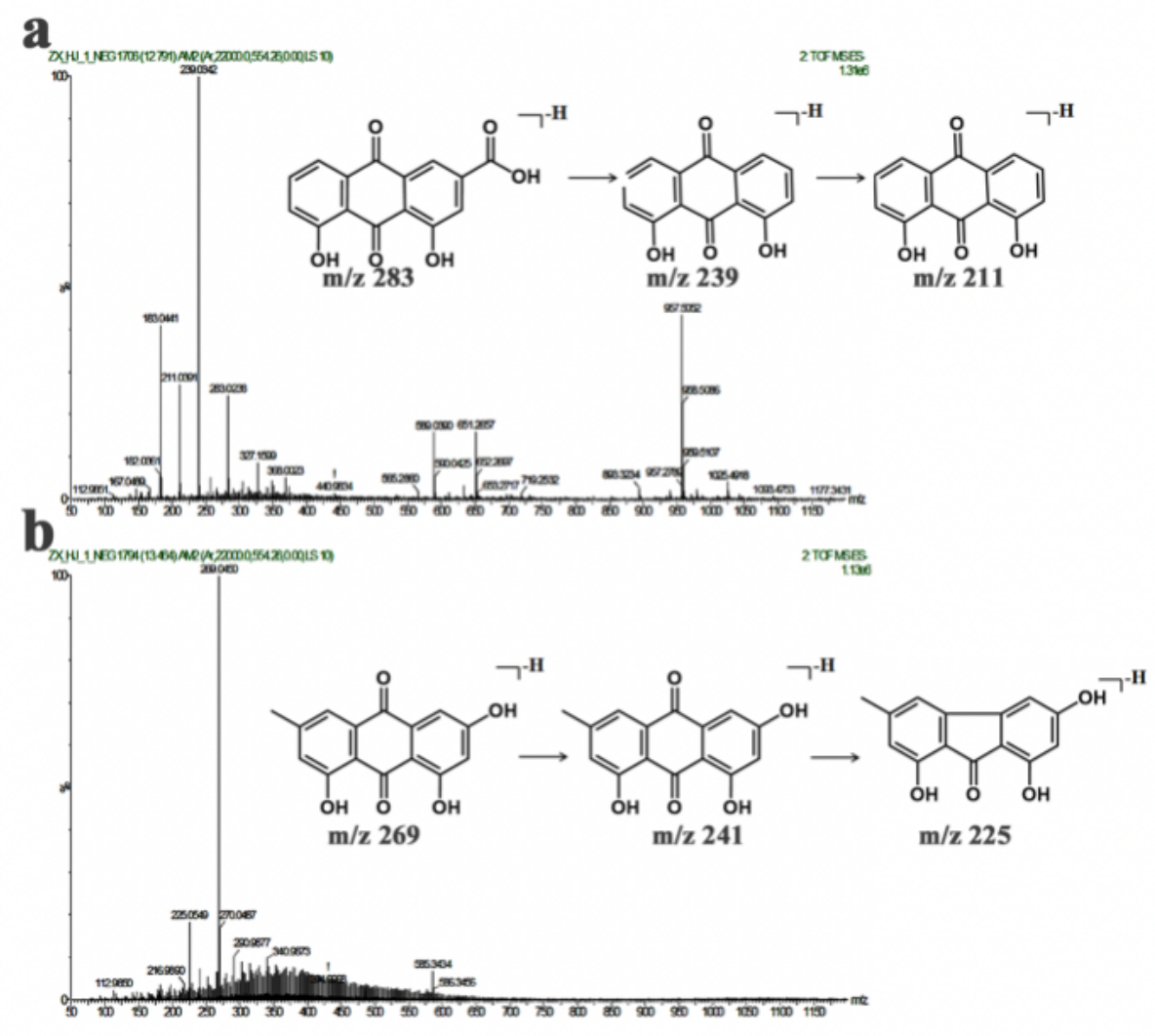

Figure 10

Fig. A3 The MS/MS spectra and proposed fragmentation pathways of rhein (a) and emodin (b) in the negative ion mode. 